\title{
Gastrointestinal Manifestations of Rheumatic Diseases
}

\author{
Hussein Halabi, Ammar AIDabbagh, \\ and Amany Alamoudi
}

\subsection{Objectives}

- To describe gastrointestinal manifestations in rheumatic diseases.

- To construct a diagnostic and systemic approach to gastrointestinal symptoms in rheumatic diseases.

- To interpret laboratory, radiological, and endoscopic finding in patients with rheumatic diseases presenting with gastrointestinal manifestations.

\subsection{Gastrointestinal Manifestations of Systemic Lupus Erythematosus (SLE)}

SLE may involve any part of the gastrointestinal (GI) tract as well as the liver.

H. Halabi

Department of Internal Medicine, King Faisal Specialist Hospital and Research Center, Jeddah, Saudi Arabia

A. AlDabbagh $(\bowtie)$ Internal Medicine, Gastroenterology and Hepatology Consultant, Jeddah, Saudi Arabia e-mail: aaldabbagh@fakeeh.care
A. Alamoudi
Doctor Soliman Fakeeh Hospita, Jeddah,
Saudi Arabia

Some patients may develop GI manifestation at onset of the disease (42\%), which may delay the diagnosis of SLE in those patients. Patients with SLE who present with GI symptoms may have these symptoms secondary to active disease, side effects with medications, or secondary to infectious process. The most common symptoms are nausea and vomiting (53\%), anorexia (49\%), and abdominal pain (19\%) [1]. The prognosis in such cases depends on early recognition and proper management [2].

\subsubsection{Oral Cavity Manifestations of SLE}

Oral cavity manifestations can happen in $7-52 \%$ of SLE patients and are necessarily associated with disease activity. The abnormalities which are secondary to active lupus are usually erythematosus, discoid, and ulcerative and can be painful or painless [3].

There are no high-quality evidences to guide in the management of oral lupus lesions using systemic therapy.

Antimalarials, azathioprine, and corticosteroid are frequently used for the treatment of severe cases. Furthermore, thalidomide and cyclosporine are commonly used as alternative therapy as shown in some studies from Europe [3] (Fig. 23.1). 


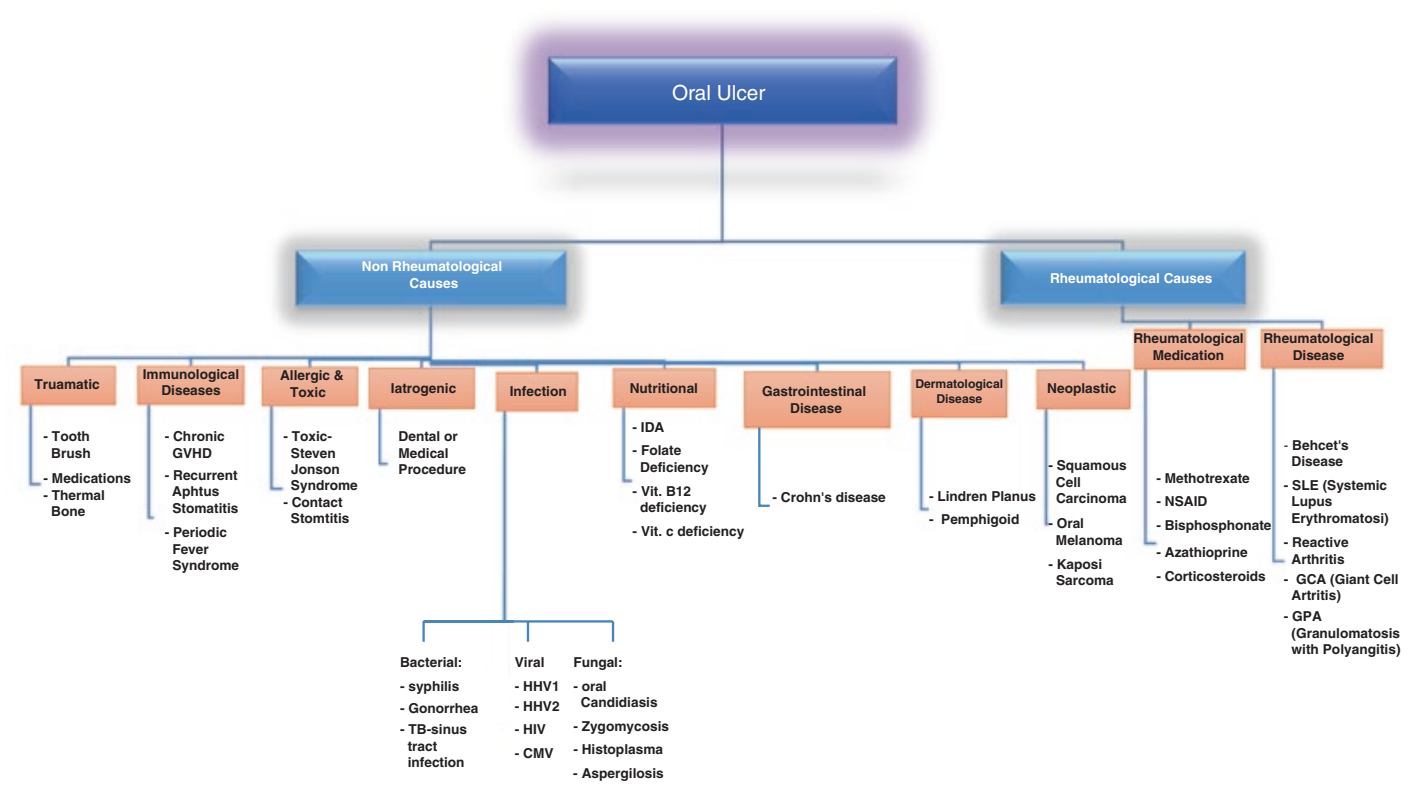

Fig. 23.1 Causes of oral ulcers

\subsubsection{Esophageal Manifestations of SLE}

Dysphagia, heartburn, and regurgitation are common among SLE patients.

Dysphagia may result from dysmotility disorder, heartburn, or reduced saliva production in case the patient has a secondary Sjogren's syndrome. The esophageal dysmotility may present in $21 \%$ to $72 \%$ of SLE patients [1].

Multiple factors may play a role in the motility changes which include inflammatory process in esophageal muscles, muscle atrophy, or ischemic vasculitis. Motility abnormalities may not be correlated to the symptoms or lupus [1]. SLE patients are at an increased risk of developing infectious esophagitis secondary to immunosuppression and pill-induced esophagitis. There are no high-quality evidences guiding the management of dysphagia and reflux in patients with SLE. Pharmacological agents, such as antacids, proton pump inhibitors, H2 blockers, or promotility agents, may play a therapeutic role [3].

\subsubsection{Gastric Manifestations of SLE}

In SLE patients who present with acute abdominal pain, perforating peptic ulcer may happen in
$6 \%$ to $8 \%$ out of them. Physicians should consider H. pylori testing before initiating treatment with NSAIDs. Additionally, those patients may require to be on continuous gastroprotective agents such as proton pump inhibitors and $\mathrm{H} 2$ blocker.

\subsubsection{Colonic and Small Bowel Manifestations of SLE}

It is always challenging for physicians if SLE patients present with acute abdominal pain. The majority of SLE patients are taking corticosteroid and/or other immunosuppressive medications, which could mask some clinical signs of perforation and ischemia [3].

The most prevalent etiologies of acute abdominal pain in patients with SLE are mesenteric vasculitis, hepatobiliary disease, pancreatitis, gastroenteritis, and appendicitis. Acute abdominal pain in SLE patients is associated with relatively high mortality reaching $9.4 \%$ to $11 \%$ [1].

During flare of SLE, $53 \%$ of the patients who present with acute abdominal pain may have intestinal vasculitis, and their initial symptoms may include acute abdominal pain, nausea, and vomiting.

Intestinal vasculitis may lead to ischemic changes and infarction, and its affection may 
range from superficial ulcerations to deeper layers which may cause penetration of the submucosa; in some cases GI bleeding may happen.

There are certain clinical and radiological signs that may alert physician for the possibility of bowel perforation which include low blood pressure, metabolic acidosis, high levels of serum lactate dehydrogenase, distended abdomen, and dilated intestines in abdominal radiograph.

Thumb printing represents edema of the submucosa or bleeding on a barium enema; this sign is considered specific for intestinal ischemia [3]. Colonoscopic findings may reveal inflammatory changes and/or ulcers, which may be irregular in shape or punched out. Threephase abdominal Tc-99 m pyrophosphate scintigraphy can be utilized to show areas of active vasculitis. Mesenteric angiography may be used to rule out distinct type of vasculitis like polyarteritis nodosa which may be associated with diffuse irregularities of the small arteries in the intestine and renal arteries [1].

Treatment of lupus vasculitis includes treatment of underlying SLE and high doses of steroids, but in advanced cases, cyclophosphamide is usually given. Patient must be referred to general surgery because they should have a low threshold for early laparotomy in cases of acute abdomen [1].

In SLE patients, infection is considered one of the most common causes of mortality which could reach up to $28.5 \%$ [1, 3]. The majority of SLE patients are considered immunocompromised because of immune dysregulation associated with the disease itself and because most of the patient are being managed with immunosuppressant medications. Thus, they are at risk of certain kinds of infections such as CMV, Salmonella infection, pneumatosis cystoides intestinalis, and others.

In infection with Salmonella, the bacteria is usually isolated from blood rather than the stool sample. It is usually associated with febrile illness and abdominal pain and infrequently diarrhea [1].

Patients with pneumatosis cystoides intestinalis could have benign pneumoperitoneum. Conservative management may be sufficient, and some patients may require corticosteroids or intravenous cyclophosphamide [1].

Because of the similarity between clinical symptoms of SLE flares and those associated with infections, early identifications of underlying infections among SLE patients can be challenging [3].

One of the rare GI complications in patients with SLE is intestinal pseudo-obstruction (IPO), and it is defined as having symptoms and signs of intestinal obstruction with the absence of actual mechanic obstruction.

IPO can be diagnosed based on the clinical findings, abdominal radiograph, and manometry findings [4]. Management usually starts with conservative measures; in case of insufficient response, steroids and/or immunosuppressive drugs can be given as well as antibiotics specially the ones with prokinetic properties such as erythromycin. Octreotide may be used in refractory cases [1].

Protein-losing gastroenteropathy (PLE) is described as the presence of low levels of serum albumin secondary to losing proteins from the GI tract, and it's usually considered in patients with hypoalbuminemia with the absence of marked proteinuria, advanced hepatic disorders, impaired absorption, or poor oral intake [4]. The small intestines are commonly affected rather than the large intestines [1]. It commonly manifested as mild edema and may progress to ascites and pleural and pericardial effusions [4]. Radiological features of PLE may include prominent mucosal pattern (due to edema), speculation, and fragmentation or clumping of barium. Histological features may be normal or may show blunted villi or lymphangiectasia. Management of PLE includes corticosteroids, and some patients may require the use of cytotoxic medications; octreotide is occasionally used [1].

\subsubsection{Pancreatic and Gallbladder Manifestations of SLE}

Acute pancreatitis is an uncommon complication of SLE, and it might occur in $8 \%$ of patients with SLE, and patient may develop abdominal pain [1]. 
SLE patients with pancreatitis have a mortality rate of $27 \%$. It is associated specially with neuropsychiatric and cardiac manifestations, hypocalcemia, low levels of the complements, and complications of pancreatitis [1]. The management of SLE-related pancreatitis, as with other causes of acute pancreatitis, is with intravenous fluids, pain control, and electrolyte correction.

Primary sclerosing cholangitis and autoimmune cholangiopathy have been reported in SLE patients. Acute acalculous cholecystitis may occur secondary to vasculitis or serositis, and it is commonly managed with surgical intervention [1] (Fig. 23.2).

\subsubsection{Hepatic Manifestations of SLE}

It was estimated in some studies after several years of follow-up periods that $9.3 \%$ to $59.7 \%$ of
SLE patients may have an abnormal liver function test (LFT) [5]. Transaminitis is common among SLE patients and may be developed due to several etiologies which may include side effects of medications, infectious viral hepatitis, fatty liver disease associated with steroid use, hepatic congestion, primary liver disease, autoimmune hepatitis (lupoid hepatitis), or lupus hepatitis [6]. During SLE flare, 20\% of the patients may have abnormal liver enzymes, while in $23 \%$ of the patients, the etiology of the abnormal liver tests is unknown [7]. SLE patients may have hepatomegaly in $39 \%$ to $40 \%$ of the cases, while splenomegaly may present in $6 \%$ of patients with SLE [1].

Autoimmune hepatitis (AIH) should be always considered in SLE patient with persistent elevated liver enzymes; undiagnosed AIH may progress rapidly to hepatic cirrhosis. Both diseases are associated with positive ANA; however, anti-smooth muscle antibody is associated

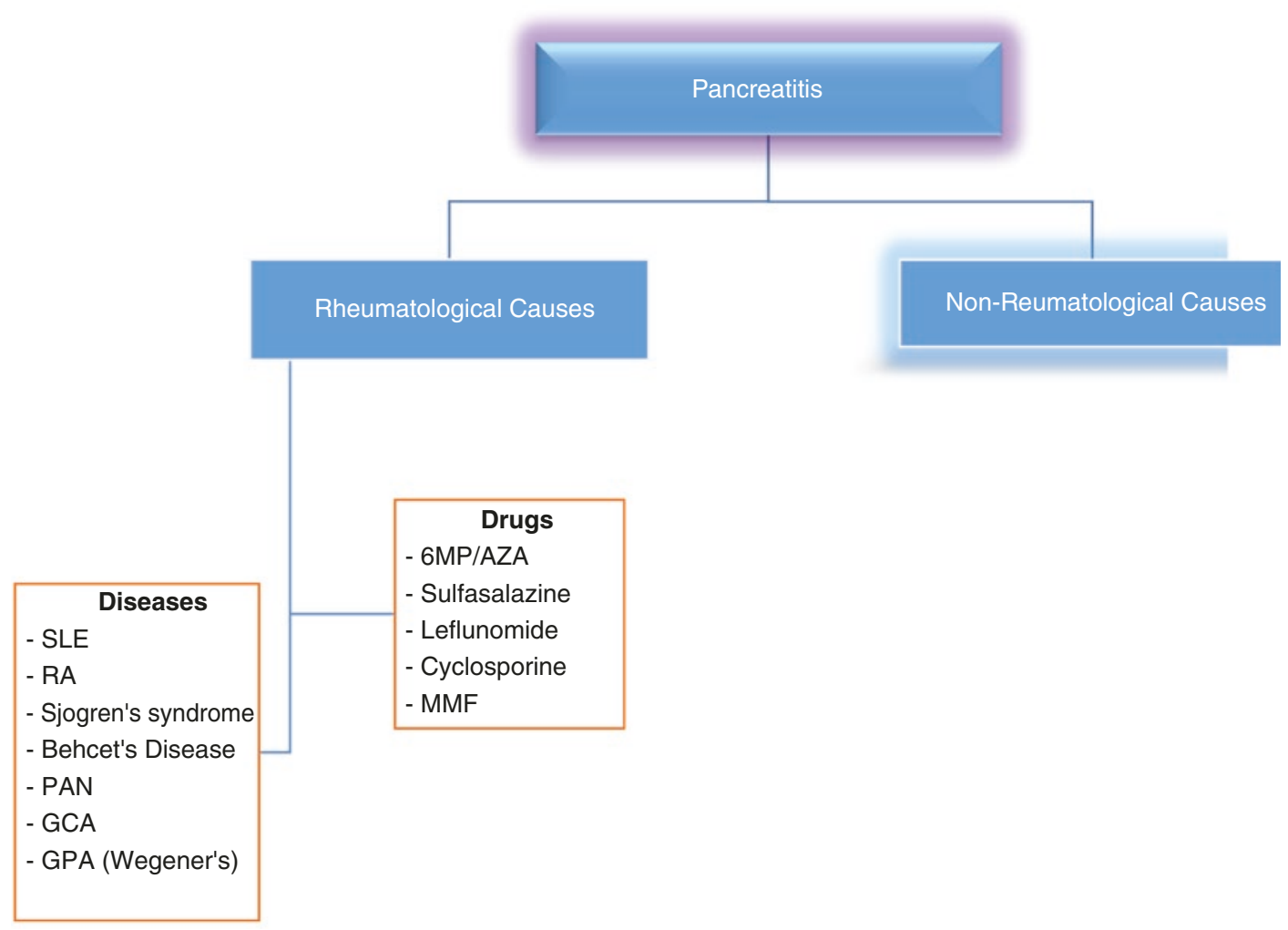

Fig. 23.2 Rheumatological causes of pancreatitis 
with AIH and can help in distinguishing between AIH and lupus-associated hepatitis. On the other hand, anti-ribosomal $\mathrm{P}$ antibody was found to be positive in many patients (69\%) with lupus hepatitis [7].

Patients with AIH are usually managed with corticosteroid initially, and some of them require other immunosuppressant medications [8].

Based on the current understanding of lupoid hepatitis, it can be defined as the presence of pathologic liver injury fitting a picture of chronic hepatitis, having a negative serum serology of the common viral infections associated with chronic hepatitis and the presence of positive ANA or LE cell preparation [9].

Luckily, there are some histological findings seen in liver biopsies which may be helpful for distinguishing lupus hepatitis from AIH. For instances, in $\mathrm{AIH}$, interface hepatitis associated with lobular activity, rosetting of liver cells, or lymphoplasma cell infiltration can be seen in liver biopsy. On the other hand, in lupus hepatitis, the inflammation is usually lobular and occasionally periportal, with a paucity of lymphoid infiltrate [6].

SLE patient may have a secondary antiphospholipid syndrome (APS), which is described as the presence of antiphospholipid antibodies and recurrent arterial or venous thrombosis. Patients with APS have GI involvement such as BuddChiari syndrome, hepatic ischemia, and esophageal varices (secondary to portal vein thrombosis); esophageal involvement may occur as well which include necrosis with perforation (due to thrombosis), bowel ischemia, colonic ulcers, and pancreatitis [10].

Overlap syndrome can occur in which patient may have SLE with AIH or PBC with similar prevalence $(2.7 \%$ to $15 \%)[7,11]$.

\subsubsection{Gastrointestinal Malignancies in Systemic Lupus Erythematosus}

Several studies have looked at the malignancy rate among SLE patients. An international study which involved 16,409 SLE patients noted a modest increased risk of malignancy in SLE patients [12]. Multiple factors are possibly contributing to this slight increase of overall malignancy risk in those patients which include immune dysregulation in SLE which may lead to disturbance in abnormal proliferations and activation of $\mathrm{T}$ and $\mathrm{B}$ cells. Thus, abnormality in $\mathrm{B}$ cell proliferation may explain the presence of non-Hodgkin's lymphoma, a type of B cell lymphoma, in some SLE patients. Other factors are the use of immunosuppressive medications as well as the chronic inflammation associated with the disease itself [13].

The most frequently noted malignancies in SLE patients are non-Hodgkin's lymphoma, lung cancer, hepatobiliary malignancy, vulvar/vaginal malignancy, and thyroid malignancies, as well as cervical dysplasia [12].

On the other hand, there are some malignancies found to be decreased among patients with SLE such as breast and prostate cancer. Some proposed reason for this decrease in the rate of these malignancies may be related to circulating anti-DNA autoantibodies as well as certain cytokines mediated by HSP-27 [12].

\subsection{Gastrointestinal Manifestations of Rheumatoid Arthritis (RA)}

\subsubsection{Dysphagia and Other Esophageal Manifestations of Rheumatoid Arthritis}

Almost half of RA patients may have temporomandibular joint (TMJ) arthritis. Patients with TMJ arthritis may complain of pain and crepitus during chewing secondary to TMJ involvement which may correlate with RA activity. Atlantoaxial subluxation with evidences of spinal cord involvement may result in dysphagia; physicians should be aware of the high risk associated with endoscopy in such patients. Patients with juvenile RA (JRA) may complain of dysphagia secondary to cervical spine abnormality or to micrognathia, which occurs as a result of the loss of the mandibular condyles and retraction of the jaw [14]. Methotrexate, the cornerstone of treat- 
ment of RA, can cause oral ulcers which might contribute to the difficulty in initiating swallowing or dysphagia.

Esophageal manifestations in RA may include esophageal dysmotility, reflux esophagitis, amyloidosis, and, rarely, esophageal varices due to Felty's syndrome.

Abnormal esophageal motility with a low peristaltic pressure in the lower two-thirds of the esophagus and reduced pressure in the lower esophageal sphincter lead to impaired peristalsis in patients with RA. These manifestations may occur in up to $62.5 \%$ of RA patients and can be associated with heartburn, dysphagia, and esophagitis. Esophageal dysmotility may be attributed to amyloidosis or to GI vasculitis, which occasionally can cause esophageal strictures from local ischemia [10, 14].

\subsubsection{Gastric Manifestations of Rheumatoid Arthritis}

Most of GI abnormalities in patient with RA are associated with the chronic use of NSAIDs and steroids. In $20 \%$ to $40 \%$ of the patients on NSAIDs, abnormal changes can be seen during endoscopic evaluations. Those patients are considered at a high risk of peptic ulcer disease and ulcerations in both small and large bowels [10].

In $30 \%$ and $60 \%$ of patients with RA, biopsy samples may show chronic superficial and atrophic gastritis. In addition, chronic atrophic gastritis can also be seen in patients with RA and associated secondary Sjogren's syndrome. Those patients may develop vitamin B12 deficiency and/or pernicious anemia [14]. NSAIDs are commonly prescribed for RA patient because of their effect for pain relief and for their antiinflammatory properties; however, their use is associated with a wide range of GI manifestations, and patients may present with relatively mild symptoms such as dyspepsia and gastroduodenal ulcerations to a life-threatening ones such as GI bleeding, perforations, or obstructions. Gastropathy associated with NSAID use may direct physicians toward the use of a selective type of NSAIDs known as cyclooxygenase 2 inhibitors (COX-2), which in many trials proven its efficacy in reducing GI complications such as bleeding, perforations, or obstructions. Physicians may use alternative methods for GI protection with NSAID use such as prescribing proton pump inhibitor (PPI) or misoprostol combined with NSAIDs $[15,16]$.

\subsubsection{Intestinal and Colonic Manifestations of Rheumatoid Arthritis}

Small bowel findings in patients with RA may manifest as inflammatory changes which could result in blood and protein loss, ulcerations, and strictures. However, colonic and rectal involvement includes nonspecific colitis and rectitis, ulcerations, blood loss, diverticular complications, and perforation. The cecum and the right colon are the common sites of colon ulcerations which may complicate with a bleeding or perforations [10].

RA-associated vasculitis is an uncommon complication of RA; it can happen in $1 \%$ of the patients. Among RA patients with associated vasculitis, $20 \%$ of them may develop intestinal involvement. Patients at risk of RA vasculitis are those with long-standing erosive arthritis, positive rheumatoid factor with a high titer, and the presence of subcutaneous nodules.

Surprisingly, RA vasculitis may occur in patients with inactive joint disease. Furthermore, it can complicate with GI bleeding, ulcerations, bowel perforations, and small and large intestine infarctions [10, 15]. The prognosis in such patients is commonly poor, and the consequences may be fatal [17].

Long-standing RA may complicate with secondary amyloidosis which involves the GI tract as well as the liver. GI involvement may manifest as protein-losing enteropathy, colon ulcers, or esophageal strictures [18].

Pneumatosis cystoides intestinalis is rarely associated with RA [19]. GI side effects associated with use of NSAIDs do not merely involve the upper GI tract; it also can involve the lower GI tract [15]. 
Anti-gliadin IgG may be found in up to $47 \%$ of patients, specially in those with positive rheumatoid factor (IgA). Duodenal villous atrophy may present in some patients; however there are insufficient evidences to support the association between RA and celiac disease.

\subsubsection{Hepatic Manifestations of Rheumatoid Arthritis}

Enlarged liver may be seen in up to $22 \%$ of RA patients using scintigraphy scan, and it may be associated with elevated RF.

Spontaneous rupture of the spleen may occur with or without splenomegaly. RA may involve the capsule which could lead to this complication. Regarding laboratory investigations, patients with RA usually have normal levels of transaminases and bilirubin; however, alkaline phosphatase may be elevated in up to $18 \%-46 \%$, while gamma glutamyl transaminase (GGT) may be elevated in $23 \%-77 \%$ of RA patient and it may correlate with RA activity.

In autopsy study, abnormal liver histology was found to be in $92 \%$ of RA patients and $65 \%$ of patients in a clinical study. The most prevalent histologic findings are periportal fibrosis, inflammatory changes in the portal tract, sinusoidal dilatation, amyloid, and rarely cirrhosis. These changes are usually mild and might correlate with RA activity.

RA has been commonly reported after an infection with hepatitis B or C. However, it is still unclear as to whether the virus triggers RA or the infections and RA occur at the same time. After following levels of $\mathrm{HCV}$ viral loads and liver function tests, it was found that the use of antiTNF biological therapy in the treatment of RA may not cause a reactivation of chronic infection with HCV. On the other hand, anti-TNF may cause a reactivation of HBV. It is recommended for all patients with $\mathrm{HBV}$ who are planning to get anti-TNF to be started on the treatment for HBV at least 2 weeks before the initiation of anti-TNF.

In RA patients presenting with liver abnormalities, physicians should broaden the differen- tial diagnosis to include side effects of a drug with hepatotoxicity, viral hepatitis, fatty liver, and autoimmune hepatitis (AIH).

Sulfasalazine can cause reversible liver injury but might recur if the drug was reintroduced.

Methotrexate hepatotoxicity was extensively reviewed and can cause steatosis, stellate cell hypertrophy, and hepatic fibrosis. Hepatic damage may increase with recurrent hepatic infections and concomitant use of the hepatotoxic drugs or alcohol [20].

\subsubsection{Other Gastrointestinal Manifestations of Rheumatoid Arthritis}

Rheumatoid vasculitis is classified as vasculitis associated with a systemic disease. It usually involves small- and medium-sized vessels affecting $1-5 \%$ of RA patients.

Intestinal involvement in rheumatoid vasculitis was described in another section (see Intestinal and Colonic Manifestations of Rheumatoid Arthritis).

In case of ruptured aneurysm, patients may develop abdominal pain and syncope. Regarding hepatic manifestations, rheumatoid vasculitis may lead to intrahepatic or subcapsular hematomas, infarction, or rupture [10, 21]. Management of rheumatoid vasculitis is based on only small observational studies and case reports.

The most commonly used agents are highdose steroids, cyclophosphamide, and biological therapies.

Secondary amyloidosis can be caused by several diseases; however, RA is the most common cause of secondary amyloidosis. Patients at risk are those with poorly controlled and longstanding disease usually more than 5 years. Secondary amyloidosis may involve the GI tract in up to $22 \%$ of the cases. It may manifest as refractory diarrhea, malabsorption, proteinlosing enteropathy, and abdominal pain [22-24].

Presence of splenomegaly, neutropenia, and RA makes the classic trial of Felty's syndrome which may present in $1 \%$ of RA patients. It is characterized by severe destructive arthritis, 
rheumatoid nodules, enlarged lymph nodes, vasculopathy, skin ulcers, and hepatic abnormality which may include hepatomegaly in up to $68 \%$ of the cases and abnormal liver function tests in up to $56 \%$ of the patients which is a higher percentage compared to the one seen in uncomplicated RA.

\subsection{Gastrointestinal Manifestations of Inflammatory Myositis}

Inflammatory myopathies are distinct category of rheumatic diseases which usually present with a proximal myopathy; however they have different skeletal and other organ manifestations; dermatomyositis (DM), polymyositis (PM), and inclusion body myositis (IBM) are the main diseases in this category.

\subsubsection{Symptomatology}

Gastrointestinal manifestations of inflammatory myopathies include dysphagia, heartburn, bloating, nausea, and chronic constipation. Severe GI manifestations maybe secondary to inflammatory changes in bowel mucosa, resulting in erosions, ulcers, and perforations, are uncommonly seen in adult DM $[25,26]$.

\subsubsection{Esophageal Manifestations of Inflammatory Myositis}

Patients with inflammatory myopathies and esophageal involvement may develop uncoordinated swallowing, uncoordinated esophageal peristalsis, and hiatus hernia with reflux and stricture formation [27].

The most common GI symptoms in patients with inflammatory myositis are dysphagia-to solids and liquids - and heartburns [25]. They occur secondary to abnormalities in the pharynx and esophagus and in up to $32-84 \%$ of patients with myositis. The highest type of inflammatory myositis in the percentage is inclusion body myositis, and it is unfortunately the most refractory one [28]. The patient might present with symptoms including nasal speech, hoarseness of the voice, nasal regurgitation, and an inability to swallow a food bolus while the patient is on recumbent position due to the elimination of the effect of gravity; physical exam will be significant for tongue weakness, flaccid vocal cords, and poor palatal motion $[27,28]$. The presence of esophageal manifestations is linked to unfavorable prognosis and a more severe disease.

Patients with reflux symptoms might respond to anti-reflux measures as well as treatment of inflammatory myopathy [25]. The use of steroids may improve esophageal dysfunction. In PM and DM, plasmapheresis may be effective for the treatment of dysphagia [29]. Intravenous immunoglobulin (IVIG) is usually considered in the refractory inflammatory myopathy, with consistent remission maintained in almost half of successfully treated patients with PM after discontinuation of therapy [30]. In IBM, IVIG may be effective as well if given with or without steroid in cases of severe dysphagia. Surgical intervention is usually needed in case of obstructive causes.

Cricopharyngeal myotomy is the most beneficial intervention for dysphagia in inflammatory myopathy [28], but dilatation may be attempted if surgery is contraindicated. Injection of botulinum toxin A into the cricopharyngeus may also eliminate the need for surgical myotomy [27, 29-31].

\subsubsection{Gastric Manifestations of Inflammatory Myositis}

Esophageal as well as gastric emptying can be delayed in PM and DM. Manometry may reveal reduced distal esophageal/gastric emptying implying malfunction of the smooth muscle of the upper GI tract [25]. Delayed gastric emptying, constipation, and boating all are common in patient with inflammatory myopathy which can be attributed to dysmotility disorders. Patients with inflammatory myopathies (6-60\%)-specially DM-are at high risk of certain types of 
malignancy [10]. There is a threefold increased risk for cancer of the stomach, pancreas, and colon [32]. The most common GI malignancies are gastric and colorectal adenocarcinoma [10].

\subsubsection{Intestinal Manifestations of Inflammatory Myositis}

Vasculitis may lead to ulcerations in the mucosa and possibly intestinal perforation. This is more common in childhood type of DM rather than adult type, in which all these features have been described throughout the GI tract from the esophagus to the large intestine. Pneumatosis cystoides intestinalis has been reported in DM and PM. There have been also some reports linking between PM and small bowel pseudo-obstruction and pseudomonal necrotizing enterocolitis [25] (Fig. 23.3).

\subsubsection{Hepatic Manifestations of Inflammatory Myositis}

Inflammatory myositis is occasionally misdiagnosed as a liver abnormality. Thus, this could result in a delay in delivering the appropriate management. Active muscle inflammation is commonly associated with an elevation of the levels of CK, aldolase, ALT, AST, and LDH. Higher levels of these enzymes are commonly seen in PM compared to DM and in males compared to females. Liver abnormality or biliary diseases should be suspected in case the levels of transaminases are higher than CK levels or when patients develop cholestatic picture [33] [9].

There are some case reports associating between PM and primary biliary cirrhosis (PBC); physicians may pay attention for patients with elevated alkaline phosphatase in light of the possible association [9].

\subsection{Gastrointestinal Manifestations of Systemic Sclerosis}

Gastrointestinal manifestations of systemic sclerosis are common, and they have an effect on prognosis, morbidity, mortality, and quality of life. They result from fibrosis and can affect several portions of the GI tract. GI involvement is the most frequent internal complication and accounts for about $10 \%$ of the presenting features in systemic sclerosis [34]. Furthermore, it is possibly the second most prevalent site of systemic sclerosis visceral damage [35]. In systemic sclerosis, women are affected 4.6 times more than men [36].The pathophysiology of SSc of the GIT is known only to a limited extent. It is due to fibrotic changes caused by an increase in the collagen deposition and other extracellular matrix components in the upper and lower GIT leading to dysmotility, malabsorption, malnutrition and

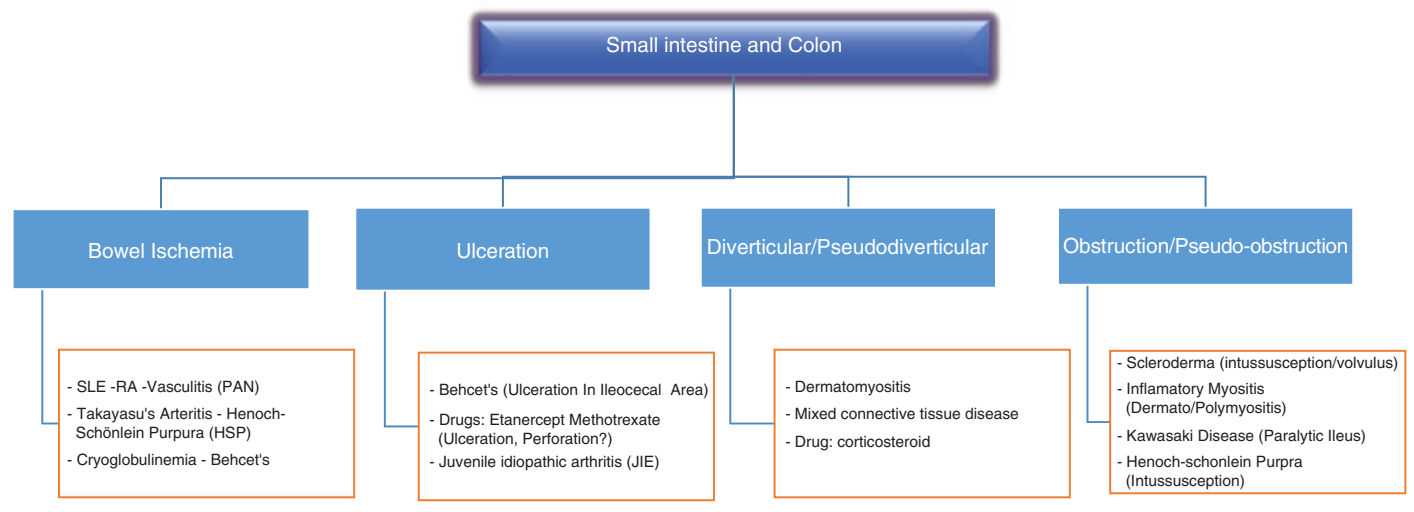

Fig. 23.3 Colonic and small intestinal manifestations of rheumatological diseases 
dilation of the intestine [36], and alteration of the microvasculature, the autonomic nervous system, and the immune system. Sjogrin proposed a progression of sclerodermatous GI involvement: vascular damage (grade 0), neurogenic impairment (grade1), and myogenic dysfunction (grade2) with the replacement of normal smooth muscle by collagenous fibrosis and atrophy [35]. The most common organ involved in GI manifestation is the esophagus, followed by the anorectum and small bowel [34], but any portion of the tact can be involved in both limited and diffused SSc. Physicians should have a high level of suspicion for GI abnormality in SSc, because patients with SSc may have subclinical GI abnormalities, in $50 \%$ of patients with esophageal involvement and $20 \%$ of small intestine involvement [2]. The mortality rate secondary to GI complications is estimated in $6-12 \%$ of the cases [35].

The oropharyngeal involvement in patients with systemic sclerosis includes skin thickening, xerostomia, and swallowing difficulties (Fig. 23.4).

\subsubsection{Esophageal Manifestations of Systemic Sclerosis}

Esophageal manifestations in SSc may occur in up to 70 to $90 \%$ [2]. Asymptomatic esophageal changes may happen in $50 \%$ of the cases, Thus, early recognition is crucial in order to avoid the complications.

Esophageal manifestations of SSc may not always be symptomatic, but early diagnosis remains important as the delay may increase the risk of complications [35]. All the symptoms are related to esophageal motility disorder and gastroesophageal reflux. Symptomatic patients may complain of heartburn, dysphagia, or odynophagia and, with advanced dysphagia, may complain of food and fluid regurgitation. Gastric reflux may lead to esophageal damage through mild peptic esophagitis, and it could progress to erosions, bleeding, and prominent ulcerations.

Patients with SSc may develop esophageal stricture formation and fistulae, and an achalasialike syndrome may result into higher risk of

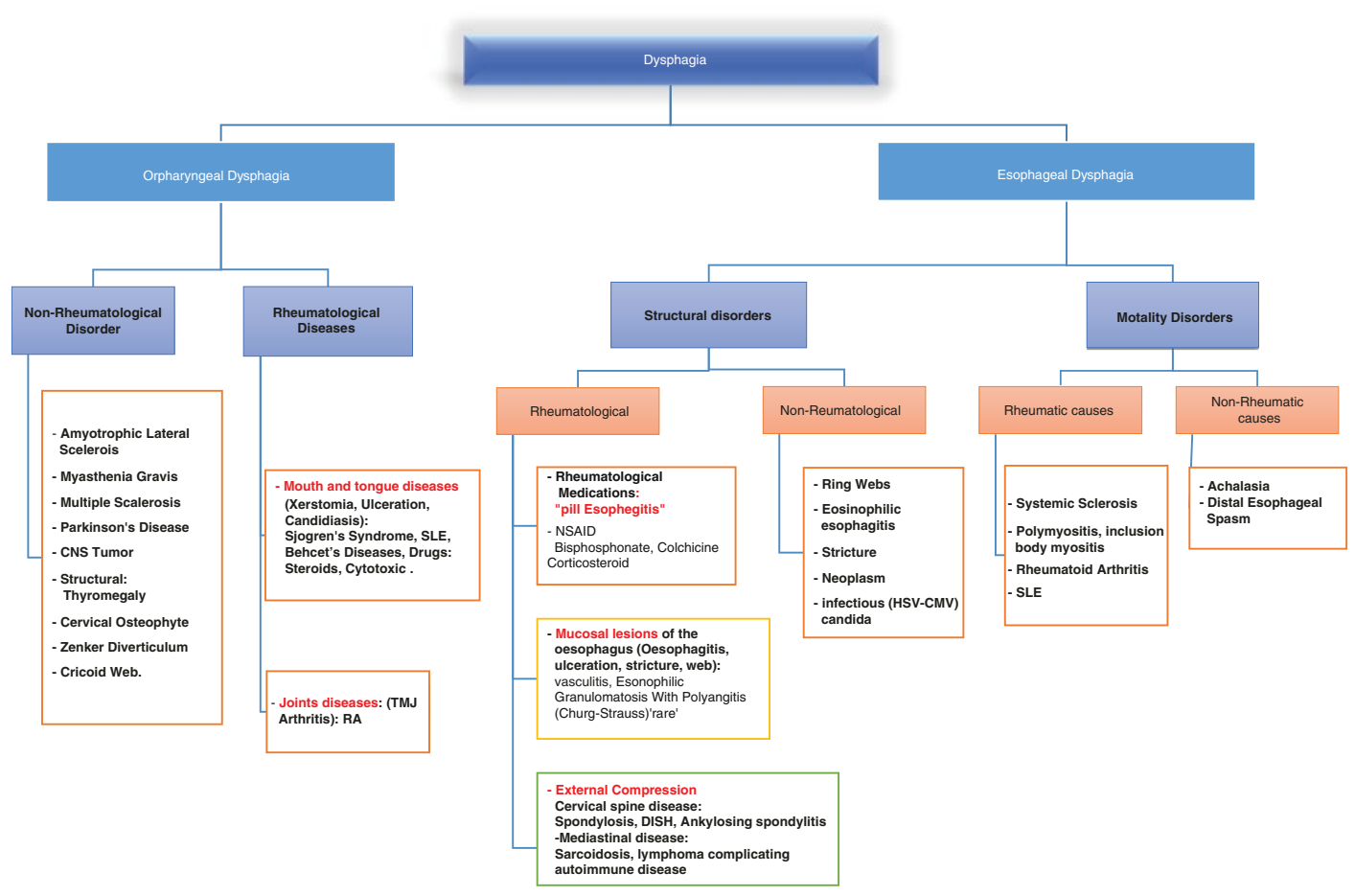

Fig. 23.4 Approach to dysphagia 
developing Barrett's esophagus [35] and esophageal adenocarcinoma [34].

Barrett's esophagus happens when the normal stratified squamous epithelial lining of the distal esophagus undergoes metaplasia, with normal epithelial cells being replaced by an abnormal columnar epithelium including goblet cells. It has an incidence rate of $6.8-12.7 \%$ compared with that of the general population (less than 1\%) [35]. Evaluation for esophageal involvement is guided by the patient's symptoms. EGD should be done in any patient with refractory heartburn, dysphagia, and odynophagia. Treatment with proton pump inhibitors for 6 months may result in complete resolution of inflammation [34].

\subsubsection{Gastric Manifestations of Systemic Sclerosis (SSc)}

Patients with SSc may have gastric involvement of the disease in 10-75\% of the cases [37]. The most common finding among these patients with $\mathrm{SSc}$ is delayed gastric emptying [2] followed by iron deficiency anemia which may be found in $96 \%$ of patients with SSc, and it is mostly due to gastric antral vascular ectasia (GAVE); it is also called watermelon stomach [37]. GAVE has been found more in patients with early diffuse cutaneous SSc and late-onset anticentromere-positive limited cutaneous SSc [37]. The diagnosis of delayed gastric emptying is made by electrogastric graphic recording or by scintigraphy following a radiolabelled meal. GAVE can be diagnosed endoscopically with two unique findings, i.e., (i) classic "watermelon stomach" with prominent, flat, or raised erythematous stripes, radiating in a spoke-like fashion from the antrum to the pylorus, and (ii) "honeycomb stomach "where a coalescence of many round angiodysplastic lesions are formed in the antrum [37]. Prokinetic agents such as metoclopramide and domperidone are commonly used because of their effect on increasing the tone of contraction of gastric muscles. Patients who have insufficient response to these medications may be offered a low dose of erythromycin [35].

In patients with GAVE, in addition to measures for correcting anemia such as blood trans- fusion if indicated and iron supplementation, endoscopic laser ablation is found to be effective in up to $75 \%$ of cases [35]. Surgical antrectomy is usually not indicated. In refractory cases intravenous cyclophosphamide has been used with successful results [34].

\subsubsection{Intestinal Manifestations of Systemic Sclerosis}

SSc can involve the small and large bowel as well, including the rectum and the anus. Small intestine manifestations, in conjunction with hypomotility, cause malabsorption contributing to an increased incidence of bacterial overgrowth and pseudo-obstruction which can lead to severe malnutrition. Colonic involvement may include diarrhea, fecal incontinence, and bleeding [35]. Another complication which is associated with morbidity and mortality among SSc patients is the malabsorption syndrome, which is also linked to high disease activity. Small bowel bacterial overgrowth can be diagnosed by a positive breath test or jejuna aspirate cultures. In addition, the basic laboratory tests should be acquired for all SSc patients, including serum hemoglobin because it could indicate the presence of vitamin B12, folic acid, or iron deficiencies. In SIBO, levels of serum folic acid may be elevated due to the synthesis of folates by bacterial flora in the intestines. Additionally, serum albumin is frequently used to look for evidences of malnutrition; however, this might not be entirely accurate, because it is a negative phase reactant, which has poor sensitivity and specificity for malnutrition. Carotene levels in the serum may be utilized to screen for fat malabsorption [34].

The diagnosis of pseudo-obstruction can be done by scintigraphy or wireless motility capsule. Dilatation of intestinal loops is the most prominent radiographic feature in SSc when absence of peristalsis affects the duodenum and proximal jejunum. Teamwork among rheumatologists, nutritionists, and gastroenterologists is crucial in SSc patients with malnutrition and complicated GI disease. In case a patient with SSc present with picture of SIBO, a trial of appropriate antibiotics should be started for 10 days, regardless of the 
result of breath testing. In patients with pseudoobstruction, metoclopramide and domperidone can be tried. Subcutaneous octreotide may be considered for patients with refractory GI symptoms. In case patients failed all to abovementioned medications, parenteral nutrition or enteral feeding through jejunostomy might be considered [34].

\subsubsection{Colonic and Anorectal Manifestations of Systemic Sclerosis}

Constipation may occur in patients with SSc during early phase of colonic disease. Colonic telangiectasia and pseudodivirticula are common incidental findings and may cause anemia. Therapeutic options during early phases of constipation include bulk-forming laxatives which also might be helpful in the management of fecal incontinence. Reduction of rectal compliance may occur secondary to the deposition of collagen; this might complicate with anismus which can manifest as diarrhea. Patient may complain of severe urgency, and incontinence could occur which is usually mildly improved in medical therapy such as loperamide [35].

The anorectum can be affected in up to $50-70 \%$ of SSc patients, and $20 \%$ of cases may complicate with fecal incontinence. A recent study confirmed the involvement of the IAS in SSc patients, finding the IAS of SSc patients to be thin and atrophic compared with that of incontinent controls. Based on this finding, the most effective management of anorectal symptoms could be with sacral neuromodulation, which may be worth considering early in patients with SSc [34].

\subsection{Gastrointestinal Manifestations of Behcet's Disease (BD)}

Gastrointestinal manifestations of Behçet's disease are of great importance because they have been associated with morbidity and mortality. They follow the development of oral ulcers by 3.5 to 6 years [38].

\subsubsection{Esophageal Manifestations of Behçet's Disease}

Esophageal manifestations in $\mathrm{BD}$ are uncommon; it may occur in $2 \%-11 \%$ of the patients [38]. When the esophagus is involved, other GI parts may be involved as well in more than $50 \%$ of cases [39]. The most frequent symptoms include retrosternal chest pain, dysphagia, odynophagia, and upper and lower GI bleeding [40, 41]. Endoscopy may show single or multiple ulcers. Several complications may happen including stenosis and perforations [42]. Esophageal varices have also been reported [43].

Esophageal dysmotility may also occur in BD. In comparison to age-matched group, patients with BD may have a significantly lower esophageal pressure and relaxation [44]. In patients with $\mathrm{BD}$, upper GI endoscopy is not routinely done; however, in some cases physicians may consider upper GI endoscopy and/or manometry for patients with symptoms suggestive of underlying esophageal abnormality.

\subsubsection{Gastric Manifestations of Behçet's Disease}

Gastric involvement in BD is uncommon. Patients may complain of abdominal pain or dyspepsia [43]. Ulcerations may be found during endoscopic evaluation; they may be isolated gastric ulcers, isolated duodenal ulcers, or mixed [45].

Uncommon findings may include Dieulafoy's lesions and gastric non-Hodgkin's lymphoma [43]. Gastroparesis has also been associated to $\mathrm{BD}$ in few case reports.

\subsubsection{Intestinal and Colonic Manifestations of Behcet's Disease}

Intestinal involvements in BD can be classified into two categories: small vessel disease in which ulcers are formed secondary to mucosal inflammation and large vessel disease which may result in bowel ischemia and infarction. 
The ileocecal area is the most common site for intestinal lesions [45]. The rectum and anus are rarely involved [46].

Typically, intestinal lesions in $\mathrm{BD}$ are described as large $(>1 \mathrm{~cm})$, round-/oval-shaped, deep ulcers in the ileocecal area [43]; this is based on the findings from a landmark study from Korea where a total of 94 patients with BD complicated with intestinal involvement were studied. Terminal ileum, ileocecal valve, and cecum were the most common sites for intestinal involvement representing $96 \%$ of the cases. The pattern of intestinal involvement includes localized single ulcer in $67 \%$ and localized multiple ulcers in $27 \%$ of the cases, while multiple segments and colonic involvement are found in only $6 \%$ of cases [47].

There are some other rare abnormalities in BD which may include the presence of strictures, formation of abscess and fistula, and bowel perforations [43]. Because the management is completely different and the presentation may be confused with BD, tuberculosis (TB) should be ruled out in patients living in geographic areas which are endemic with TB. In cases where it is difficult to differentiate between $\mathrm{BD}$ and intestinal TB, some experts recommend beginning 8-week trial of anti-tuberculosis antibiotics. Another mimicker of BD is Crohn's disease (CD), which is sometimes challenging for physicians to differentiate between the two, since both of them may present in young patients. However, colonoscopy may help reaching the diagnosis. Endoscopic findings in 235 patients with $\mathrm{CD}$ and BD were studied; the most predictive findings of BD using multivariate analysis were round-shaped ulcers, focal single and focal multiple ulcers "less than 6," and absence of cobblestone appearances [48].

In contrast, the lesions in CD are characteristically described as segmental, diffuse, and longitudinal, with the presence of cobblestone appearance. Both diseases (BD and CD) may have colitis and transmural enteritis, formation of fistulae, intestinal perforation, and GI bleeding, but if there are any signs of vasculitis in the specimen, this might indicate the presence of $\mathrm{BD}$ rather than CD [49]. One of the rare complications that may also affect the GI tract in patients with BD is AA amyloidosis, usually manifested with diarrhea and malabsorption. Involvement of the renal system with proteinuria could also occur and may progress to renal failure. In such cases, the mortality is relatively high reaching $50 \%$ [45] (Fig. 23.5).

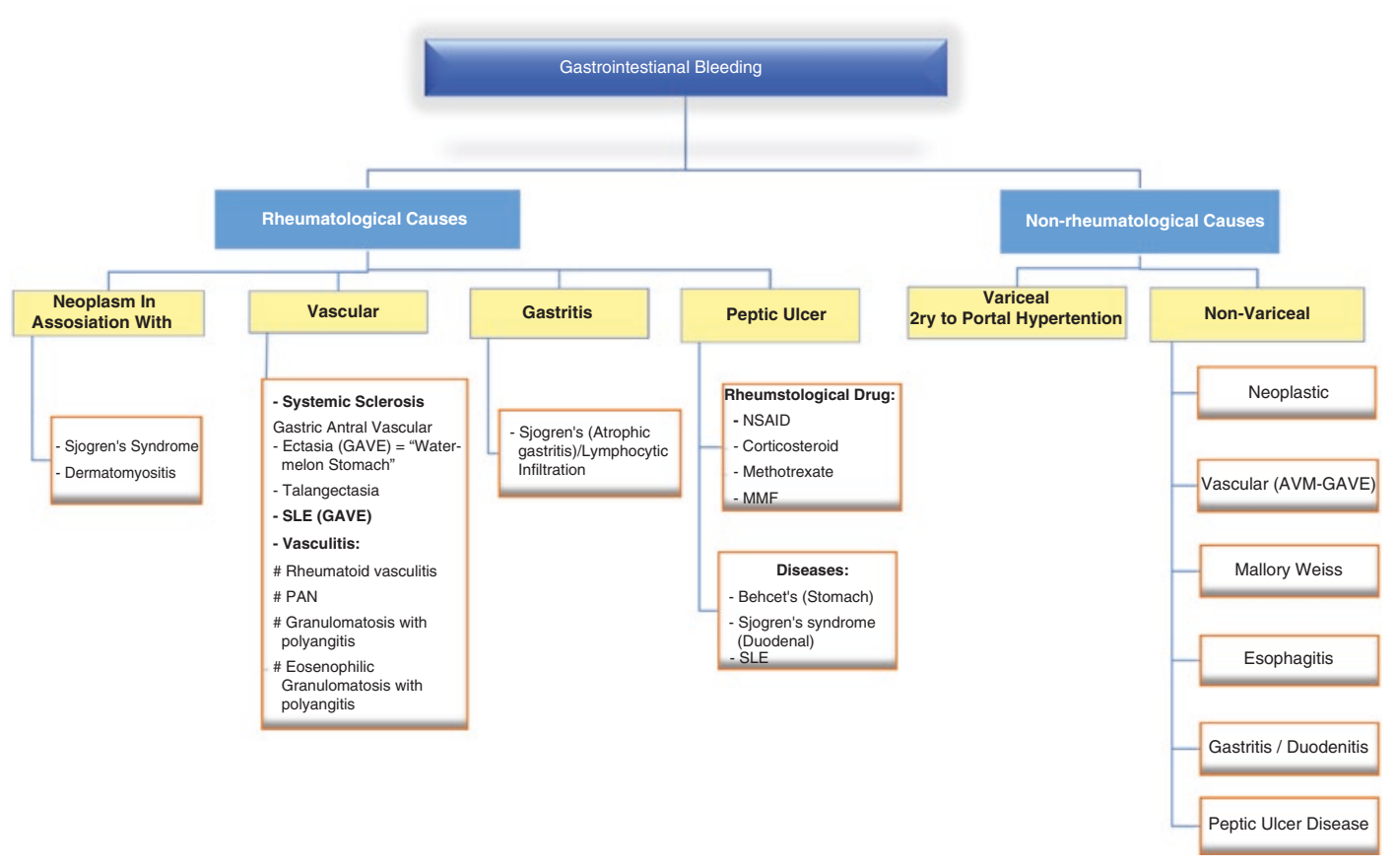

Fig. 23.5 Etiologies of gastrointestinal bleeding 


\subsubsection{Pancreatic Manifestations of Behcet's Disease}

Pancreatic manifestation in BD is very rare. Only limited case reports of acute pancreatitis have been linked to BD. Chronic pancreatitis was reported in a patient with $\mathrm{BD}$-however, this finding might be due to other etiology since the same patient was consuming high amount of alcohol $[43,50]$. Because of the lack of sufficient evidence to associate BD with pancreatitis, in a patient with BD who presents with pancreatitis, workup for other etiologies must be done [45].

\subsubsection{Hepatic Manifestations of Behcet's Disease}

Budd-Chiari syndrome (BCS) is the most common hepatic manifestation in BD. It causes high mortality rate secondary to venous thrombosis that resulted from endothelial dysfunction. Its prevalence rates are between $1.3 \%$ and $3.2 \%$ [43-45].

The patient can present right upper quadrant abdominal pain, ascites, and hepatosplenomegaly. The course of disease can be acute, subacute, or chronic [39].

Acute BCS is associated with poor prognosis due to extensive venous thrombosis.

Budd-Chiari syndrome (BCS) can be treated medically, surgically, and /or by interventional radiology. Ascites can be treated by salt restriction and diuretics. Endoscopy is indicated for screening and treatment of varices. Cyclophosphamide and corticosteroids are the cornerstone for treatment of $\mathrm{BCS}$ in patients with BD. Anticoagulation is still controversial and is not recommended in the most recent European League Against Rheumatism guidelines [39, 43].

Other hepatic manifestations of BD include liver abscess, sclerosing cholangitis, and chronic hepatitis [43].

\subsubsection{Visceral Arterial Involvement in Patients with Behcet's Disease}

BD can involve arteries and veins of all sizes. The incidence of vascular involvement is $7 \%-29 \%$ with males predominant. Arterial involvement is rare, and patients can present fever, abdominal pain, pulsatile mass, or complications such as intestinal infarction or gastrointestinal bleeding $[43,51]$.

\subsection{Gastrointestinal Manifestations of Vasculitis}

\subsubsection{Polyarteritis Nodosa (PAN)}

\subsubsection{Gastrointestinal Manifestations of Polyarteritis Nodosa}

Polyarteritis nodosa (PAN) is a necrotizing vasculitis that affects medium-sized arteries. It is associated with hepatitis B virus (HBV) in about $7 \%$ of cases [52].

Gastrointestinal manifestations occur in 14\% to $65 \%$ of patients with PAN, and it is a major cause of morbidity and mortality [53,54].

The small bowel and gallbladder are most commonly affected from the GI tract [53]. The most common symptom is chronic abdominal pain which usually develops over weeks or months [52]. The pain is usually post-prandial, and it is caused by mesenteric ischemia and can be associated with nausea, vomiting, GI bleeding, or change in bowel habits. Ischemic colitis presents with abdominal pain and bloody diarrhea. If the ischemia was limited to the mucosa; submucosa ulceration and bleeding may occur, but transmural ischemia will cause necrosis of the bowel wall which may be complicated by infarction and perforation, and this is associated with a poor prognosis [55].

Patients should be referred to surgery if they developed signs of acute abdomen in the form of rebound, rigidity, or persistent tenderness.

Multiple hemorrhagic refractory ulcers involving the stomach, duodenum, and jejunum have also been reported. Colon involvement is manifested by deep ulcers complicated by perforation or ischemic pseudomembranous colitis. Systemic vasculitis involving the appendix is also reported, and it is most often caused by PAN [56]. 


\subsubsection{Hepatic and Biliary Manifestations of Polyarteritis Nodosa}

Liver can be involved in $16 \%$ to $56 \%$ of patients [52]. Clinical manifestations of liver diseases are rare in patients with PAN. In the liver biopsy, necrotizing vasculitis may be found. Hepatic arteriograms may show caliber changes with corkscrew vessels and distal microaneurysms. If the portal vein and hepatic arteries are involved, it can lead to liver infarction, atrophy of a liver, acute liver failure, or nodular regenerative hyperplasia. Rarely, hemobilia and subcapsular or intrahepatic hemorrhage occur if aneurysmal rupture occurs in the liver.

Ascites has been reported, and it is secondary to serositis rather than to liver diseases [56-58].

Vasculitis of the arteries supplying the small bile ducts causes intrahepatic sclerosing cholangitis which is characterized by periductal inflammation, fibrous collar around the ducts, and ductal proliferation. Acalculous gangrenous cholecystitis can be a complication of arteritis as well. Biliary strictures and intracholecystic hemorrhage occur rarely from the rupture of an aneurysm of the cystic arteries into the gallbladder lumen $[52,58]$.

\subsubsection{Pancreatic Manifestations of Polyarteritis Nodosa}

The pancreatic diseases are involved in $35 \%$ to $37 \%$ of the PAN cases.

Acute pancreatitis, pseudocysts, masses, and pancreatic infarcts were reported [52].

\section{Diagnostic Modalities of Polyarteritis Nodosa}

Arteriography is the initial modality that is used in the diagnosis of PAN, and it is positive in more than $60 \%$ of patients [53]. Typical arteriographic lesions in PAN are arterial saccular and fusiform microaneurysms, which are usually associated with stenotic lesions in the kidney and mesenteric and hepatic artery branches. The diagnosis of PAN can be established when characteristic angiographic changes are detected within the appropriate clinical picture, even without histologic confirmation [59].

\section{Treatment}

Corticosteroids along with cyclophosphamide are the cornerstone of treatment for hepatitis Band C-negative PAN. Adding cyclophosphamide has shown to decrease the incidence of relapse, but it does not change the 10-year survival rate [60]. For life-threatening PAN, plasma exchanges can be used [61]. In patients with HBV-associated vasculitis, the use of anti-viral therapy in combination with corticosteroid and plasma exchanges is effective in controlling disease activity and in viral seroconversion.

The control of the viremia is also proven to help in preventing the development of cirrhosis and hepatocellular carcinoma.

Relapses are rare in HBV-related PAN and never occur when seroconversion has been achieved [62, 63].

Surgery is required for the complications, such as perforation/rupture, ischemia, or bleeding of the gastrointestinal organs or kidneys [59].

\subsubsection{Granulomatosis with Polyangiitis-GPA (Formerly Named Wegener's Granulomatosis)}

Granulomatosis with polyangiitis is manifested by necrotizing vasculitis and granulomatosis.

The disease can affect the upper and lower respiratory tract and the kidneys, with systemic involvement. GI tract can be involved in $10 \%$ to $24 \%$ of patients with GPA and is detected in an autopsy [64].

Vasculitis can cause local or diffuse pathologic changes including mesenteric ischemia, bleeding, submucosal edema, paralytic ileus, ulcerations, bowel obstruction, and perforation.

Upon studying a group of 62 patients with systemic small- and medium-sized vessel vasculitis and gastrointestinal tract manifestations, Pagnoux et al. found that the most frequent GI symptoms in patients with GPA were abdominal pain $(97 \%)$, nausea or vomiting (34\%), diarrhea (27\%), GI bleeding in the form of hematemesis $(6 \%)$, and hematochezia or melena (16\%) [65].

Any part of the GI tract could be affected. The most commonly described pathologies are ulcer- 
ations, intestinal ischemia, and perforations. Gastrointestinal manifestations can be the presenting symptom of GPA before the respiratory or renal involvement $[64,66,67]$.

\subsubsection{Eosinophilic Granulomatosis with Polyangiitis-EGPA (Formerly Named Churg- Strauss Syndrome)}

The respiratory tract is almost constantly affected, but it can be a multi-systemic disease. GI involvement can be the presenting symptoms or present concurrently with the vasculitic phase, but it has a negative prognostic factor, especially if mesenteric ischemia or bowel perforation is present [68]. GI manifestations occur in 50\% of patients with EGPA. Patients may present with nonspecific symptoms such as abdominal pain, vomiting, and diarrhea [69]. Pathologic findings include ulcers, mesenteric perforation, obstruction, and paralytic ileus with evidence of eosinophilia and granulomatosis. Because of the collateral blood supply in the GI tract, ischemic changes are rarely documented. Histologic vasculitis is rarely seen on endoscopic biopsy because submucosal vessels are too superficial to get adequate sample $[68,70]$.

Small bowel is the most commonly involved followed by the stomach and the colon [71]. Treatment of vasculitis includes steroids with cytotoxic medications for induction of remission. Depending on the degree of GI involvement, patients may require surgical interventions $[68,70]$.

\subsubsection{Henoch-Schonlein Purpura (HSP)}

Henoch-Schonlein purpura is a small-vessel IgAdominant vasculitis involving the capillaries, venules, or arterioles [72]. It can affect adults but more commonly affect children and typically after upper respiratory tract infection. GI involvement occurs in $75-85 \%$ of HSP patients $[72,73]$. The most common presenting symptom is abdominal pain and usually is peri-umbilical pain. Other GI manifestations include vomiting, GI bleeding, and paralytic ileus. Abdominal pain occurs because of extravasations of blood and fluid into the bowel wall, which can cause ulceration of the bowel mucosa and bleeding into the lumen. Upper gastrointestinal endoscopy should be considered in patients who develop GI bleeding. Fifty percent of patients may develop melena, and 15\% hematemesis [74]. Endoscopy may show hemorrhagic erosions, ulcerations, or more commonly red, small ring-like petechiae in the second part of the duodenum. Petechiae in the descending colon is commonly seen in colonoscopy [74]. Computed tomography (CT) scan may reveal wall thickening with skipped areas, mesenteric edema, and vascular engorgement [75].

Severe GI complications of HSP are rare. Intussusception secondary to submucosal hematoma is the most common ( $1 \%$ to $5 \%$ ). Intussusception should be suspected if the patient develops colicky abdominal pain that has suddenly increased in intensity and that is associated with bloody stools, and it can be diagnosed by abdominal ultrasonography $[72,74]$.

Other GI manifestations in HSP patients are protein-losing enteropathy, esophageal and ileal stricture, gastric and small bowel perforations, bowel ischemia, pancreatitis, cholecystitis, and appendicitis [73].

The prognosis for HSP is good with the exception of those patients who developed end-stage renal disease. Treatment of HSP with gastrointestinal involvement, including intussusception, bowel perforation or infarction, and severe bleeding, usually requires surgical intervention. Corticosteroids and immunosuppressive drugs can be used especially in patients with severe glomerulonephritis.

\subsubsection{Behcet's Disease}

Gastrointestinal involvement in Behcet's disease was discussed separately in a previous section of this chapter. 


\subsection{Gastrointestinal Manifestations of Spondyloarthropathies (SpA)}

Spondyloarthritis (SpA) is a group of diseases sharing the clinical, radiological, and serological manifestations in addition to having a familial and genetic link. It consists of ankylosing spondylitis (AS), reactive arthritis, psoriatic arthritis (PsA), and SpA associated with IBD (IBDassociated $\mathrm{SpA}$ ), as well as forms that do not meet the criteria of the definite categories of SpA and are thus known as undifferentiated $\mathrm{SpA}$. The incidence of SpA in patients with IBD ranges between $17 \%$ and $39 \%$. It is the most common extra-intestinal manifestation in IBD patients [76, 77]. Most importantly, the chronic type of inflammation may be considered as a risk factor for developing CD over time. Around 20\% of the patients with chronic GI inflammation on baseline ileocolonoscopy evolved into overt IBD in a 5-year period [78].

\subsubsection{Ankylosing Spondylitis (AS)}

The pathogenesis of both SpA and IBD is a result of a complex interplay between the host (genetic predisposition), the immune system, and environmental factors [78].

There is a strong genetic link between HLAB27 and AS. More than $90 \%$ of patients with AS are HLA-B27 positive. Furthermore, $25-78 \%$ of patients with IBD and AS are HLA-B27 positive. Recently, interleukin 23 receptor (IL23R) variants and the major histocompatibility complex (MHC) have been shown to be associated with AS and Crohn's disease [79].

Up to two-thirds of AS patients have subclinical GI involvement which is diagnosed either by endoscopy or histology features, and up to $5 \%-10 \%$ of cases of AS are associated with IBD [80].

Tumor necrosis factor inhibition (infliximab, adalimumab, etanercept, golimumab) can improve symptoms, signs, and the quality of life in several forms of SpA.
NSAIDs are widely used for the treatment of AS. The distal part of small bowel and colon are the major sites of side effects of NSAIDs, although the incidence of NSAID enteropathy or colopathy is lower than the upper GI tract and usually it is subclinical. However intestinal injuries induced by NSAIDs, including erosions, ulcerations, strictures, and intestinal perforations, are common. A randomized, controlled trial found that the mucosal breaks in a group who used NSAIDs plus omeprazole are more than the other group who used COX-2 inhibitors. This study showed a relative protection of using COX-2 inhibitors compared with non-selective NSAIDs plus omeprazole against small bowel injury [81]. Symptoms and signs are nonspecific such as bleeding from ulcers, anemia, hypoalbuminemia, bloody diarrhea, and signs of acute abdomen [80].

\subsubsection{Psoriatic Arthritis (PsA)}

Psoriatic arthritis (PsA) is a joint inflammation associated with skin manifestations and extraarticular involvement that affect the quality of life. PsA is considered as seronegative spondyloarthritis (SpA) [82, 83]. GI involvement such as ulcerative colitis and Crohn's disease is reported in $5-10 \%$ of the patients with subclinical course in up to two-thirds of the patients $[82,85]$. Other GI manifestations such as GERD were also reported in some retrospective studies [82].

\subsubsection{Reactive Arthritis}

Reactive arthritis can occur after an enteric infection due to Shigella, Salmonella, Yersinia, and Campylobacter species with an incidence rate ranging from $2 \%$ to $33 \%$. The presence of HLAB27 genotype and Yersinia infection increase the risk of arthritis. Arthritis develops within 2-3 weeks after diarrhea and mainly involves the knee, ankle, wrist, and sacroiliac joints. It is diagnosed based on the clinical presentation and confirmed by positive stool culture or by rising of antibody titers. 
Antibiotic treatment may be effective for diarrhea but not for arthritis [80, 84]. Steroids and azathioprine can be used in refractory cases.

\subsubsection{IBD-Associated SpA}

The onset of IBD can be preceded by SpA manifestations, but in some cases the intestinal inflammation is silent. Based on the European Spondyloarthropathy Study Group criteria, IBD is considered as a diagnostic criterion of SpA. The musculoskeletal involvement in patients with IBD can be divided into two clinical categories: axial (including sacroiliitis) and peripheral. Both categories can coexist in the same patient [85]. Other SpA manifestations such as enthesitis, tenosynovitis, dactylitis, or extra-articular manifestations (such as anterior uveitis) can also occur in IBD-associated SpA.

Axial involvement is present in $2-16 \%$ of patients with IBD and more common in Crohn's disease patients than in ulcerative colitis patients.

Axial manifestations are inflammatory back pain, isolated sacroiliitis, ankylosing spondylitis (AS), and non-radiographic spondyloarthritis. Inflammatory back pain is diagnosed clinically using the ASAS criteria. It is usually difficult to localize the pain, insidious in onset, and starts usually at rest, relieved by movement and associated with stiffness. It can be exacerbated by cough or sneezing. Patients with IBD-associated ankylosing spondylitis are found to be HLA-B27 positive in $50-80 \%$ of cases, which is less common than those with primary ankylosing spondylitis [79, 86, 87]. The clinical course is characterized by progressive spine involvement with syndesmophyte (bony growth) development and vertebral ankylosis. Isolated sacroiliitis is diagnosed by pelvic radiograph anteroposterior (AP) views or by MRI.

Physical exercise and physiotherapy have a role in maintaining the function and relieving symptoms. Medical treatment such as antiinflammatory drugs (NSAIDs/COX-2 inhibitors) are the first-line treatment for symptomatic AS; however, TNFa inhibitors (adalimumab or infliximab) are usually used as a second line of treatment when there is inadequate control of the disease by NSAID [86, 87].
The peripheral manifestations of SpA can be seen in both Crohn's disease and ulcerative colitis with prevalence rate of $0.4-34.6 \%$ of IBD patients. It is more common in individuals with Crohn's disease. Peripheral arthropathies in inflammatory bowel disease (IBD) include arthritis, dactylitis, enthesitis, and arthralgia. Peripheral arthritis can be classified as type 1 and type 2 arthritis and can coexist with axial involvement.

Type 1 is an oligoarticular ( $<5$ joints) peripheral arthritis usually affecting four or fewer joints that is usually persistent for 10 weeks and often associated with IBD relapses. In contrast, type 2 is a polyarticular arthritis ( $\geq 5$ joints) affecting five or more small joints with persistent symptoms for months to years and not associated with IBD activity [79, 84, 87, 88].

Peripheral arthropathies are diagnosed clinically; on examination, signs of active arthritis in form of swelling and pain can be found. Erythema nodosum is usually associated with the type 1 arthritis, whereas uveitis is the most common extra-articular manifestation in patients with type 2 arthritis. Laboratory tests, such as C-reactive protein (CRP) and white blood cell count, reflect the bowel activity and cannot be used as a diagnostic tool. Furthermore, peripheral arthritis is not associated with HLA-B27 positivity.

The treatment of active IBD should always bring the attention to arthropathies, which usually occur during a relapse.

The treatment includes pain management such as paracetamol, non-steroidal anti-inflammatory drugs (NSAIDs), and/or cyclooxygenase-2 (COX-2) inhibitors.

Steroid injection can be used into the affected joint. For resistant peripheral arthritis in patients with IBD, sulfasalazine and tumor necrosis factor a (TNFa) inhibitors can be used [79, 86, 87].

\subsection{Gastrointestinal Manifestations of Sjogren's Syndrome (SS)}

SS is a chronic inflammatory disorder associated with autoimmune destruction of the exocrine glands, which leads to diminished glandular secretions causing a mucosal dryness. SS is classified to either primary or secondary. The primary 
form accounts for approximately 50\% of the cases. The secondary form occurs in association with other autoimmune diseases, most commonly RA. Because of the wide distribution of exocrine glands in the GI tract, SS can involve any part of the digestive system, including the salivary glands, mouth, esophagus, stomach, pancreas, hepatobiliary organs, small bowel, and colon.

\subsubsection{Oral Manifestations of Sjogren's Syndrome}

Oral involvement in SS is characterized mainly by dryness, causing a wide spectrum of symptoms, including mild-to-severe xerostomia with dysgeusia and tooth decay. SS patients report sensitivity to acid and spicy foods, parched mouth, difficulty chewing and swallowing dry food, fissures, atrophy, and papillae of the tongue and might present with accelerated or unusual tooth decay or tooth loss, hoarseness of voice, oral candidiasis, and nasal dryness. On examination, the oral mucous membranes might appear dull, parchment-like, adhering to the examining finger. Angular cheilitis and reduction in infralingual salivary pooling could be seen. Parotid and/ or submandibular gland enlargement could be appreciated [89].

Treatment of dry mouth includes secretagogues and topical agents, which stimulate muscarinic receptors (pilocarpine and cevimeline) $[90,91]$.

\subsubsection{Esophageal Manifestations of Sjogren's Syndrome}

Dysphagia occurs in $30 \%$ to $81 \%$ of patients with SS. It is usually localized to the cervical esophagus/pharynx or midthoracic region [92].

Saliva is required for pharyngoesophageal transfer of a food bolus, and saliva reduction in SS might contribute to dysphagia. However, most authors find no relationship between dysphagia and salivary flow rates when tested and measured by the change in weight of a sponge after it is chewed [93].
Esophageal manometry is the key investigation and usually showed upper esophageal sphincter pressure. Some studies showed increased nonspecific motility abnormalities in SS $[94,95]$. During endoscopy, mucosal atrophy can be seen throughout the entire length of the esophagus.

\subsubsection{Gastric Manifestations of Sjogren's Syndrome}

Dyspepsia is found in $15.6 \%$ to $23 \%$ of patients with primary Sjogren's syndrome. Chronic atrophic gastritis was found in $25 \%$ to $81 \%$ of patients with SS who underwent endoscopy. Consistent with this finding, hypopepsinogenemia was found in $69 \%$ of patients, with half of them having elevated serum gastrin [96, 97]. One of the main concerns in SS is the increased risk of the development of lymphoma, such as mucosaassociated lymphoid tissue lymphomas (MALT lymphoma), within the GI tract. Evaluation for malignancy by endoscopic studies is crucial whenever the SS patient reported symptoms of abdominal fullness or epigastric pain [98].

Treatment of $H$. pylori does not prove to reduce gastric lymphocytic infiltration, gastric atrophy, or dyspepsia in SS. B cell clonality was noted in both the parotid and gastric tissue from six patients with primary SS and gastric MALT lymphoma [96, 97].

Chronic inflammation and/or glandular atrophy study by immunohistochemistry might reveal a predominance of CD3 + T lymphocytes, mostly CD $4+0.31$ These findings are similar to that found in the salivary glands, suggesting that $\mathrm{SS}$ is a systemic disease affecting multiple organs [98].

\subsubsection{Bowel and Colonic Manifestations of Sjogren's Syndrome}

Documented intestinal involvement is rare to absent in large series. Abdominal discomfort occurs in up to $37 \%$ of patients with SS, nausea 
$5 \%$, constipation $23 \%$, diarrhea in $9 \%$, and iron deficiency anemia due to malabsorption in up to5\% [99, 100]. Duodenal ulcers, likely related to a decrease in saliva production and duodenal gland secretion reduction, both of them have been described in SS. Celiac disease occurred in $4.5 \%$ to $15 \%$ of patients as observed in two cohorts of primary SS [101].

An occasional association of inflammatory bowel disease (IBD) with SS was suggested in case reports [102]. A study that evaluated the presence of SS among a large cohort of IBD patients found that the prevalence of SS was $4.2 \%$ to $5.7 \%$; SS was diagnosed 6 years after the diagnosis of IBD.

SS has been associated with intestinal pseudoobstruction, colon cancer, and pneumatosis cystoides intestinalis. Vasculitis in SS is rare and can be associated with cryoglobulins, and it is often life-threatening, presenting with ischemic or infarcted bowel, leading to bowel gangrene and acute surgical abdomen [103, 104] (Fig. 23.6).

\subsubsection{Pancreatic Manifestations of Sjogren's Syndrome}

Pancreatitis was documented in $7 \%$ of patients with SS. It might present as autoimmune pancreatitis or chronic pancreatitis. There are multiple reported cases of SS with pancreatic calcifications. Enlarged pancreatic head suggestive of neoplasm and increased serum CA 19-9 antibodies in benign pancreatic processes had been also reported. Pancreatic exocrine insufficiency is not uncommon, and it is related to reduced gastric secretions and/or abnormal gallbladder function.

Primary sclerosing cholangitis (PSC) and secondary sclerosing cholangitis are associated with chronic pancreatitis and SS or sicca syndrome. Treatment depends on sclerosing cholangitis status and the degree of extrahepatic involvement. Immunomodulators (including steroids, azathioprine, and rituximab) are the mainstay of treatment for autoimmune sclerosing cholangitis with or without autoimmune pancreatitis. Endoscopic treatment is directed to therapeutic intervention to release the biliary obstruction and for tissue sampling. Liver transplantation is the treatment for end-stage liver disease due to sclerosing cholangitis or recurrent cholangitis [89, 105, 106].

\subsubsection{Hepatic Manifestations of Sjogren's Syndrome}

Liver involvement is the most common nonexocrine feature in primary SS. Hepatomegaly was found in 11-21\% of patients with primary $\mathrm{SS}$ at the time of diagnosis. Abnormal liver function tests (LFTs) mainly cholestatic biochemical picture are detected in 30-60\% of cases, but hepatocellular or mixed patterns may also be observed. The most common causes of liver dis-

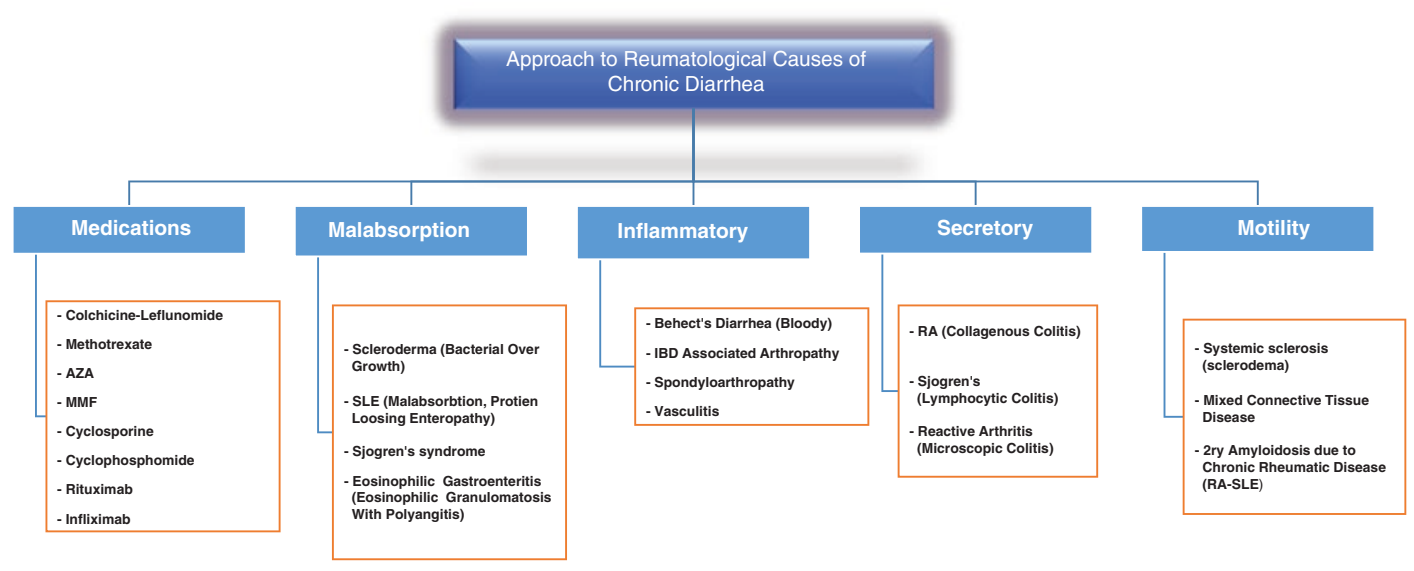

Fig. 23.6 Approach to rheumatological causes of chronic diarrhea 
ease in SS are primary biliary cholangitis (PBC), autoimmune hepatitis (AIH), nonalcoholic fatty liver disease, and HCV. AIH or PBC is associated with pSS in $50 \%$ of cases $[9,107]$.

Iron overload might cause functional damage of the salivary glands leading to sicca syndrome, which responds to iron chelation. Idiopathic granulomatous hepatitis can also occur in association with SS [108].

B cell clonality was detected in the liver of $77.8 \%$ of the patients with SS who were specifically evaluated for this. There is no increased incidence of hepatic lymphoma in SS (except for SS-HCV), suggesting that the B cell clonality is a benign antigen-driven expansion $[9,108]$.

In regard to $\mathrm{PBC}$, sicca complex is found in $35 \%$ to $77 \%$ of patients with PBC; in contrast, SS is found in $18 \%$ to $38 \%$ of patients. The degree of sicca components does not correlate with the duration or degree of liver disease or the presence of autoantibodies [107, 109].

It is worth mentioning that the salivary gland ducts of patients with $\mathrm{PBC}$-independent of the presence of sicca symptoms-manifest a PBClike immunohistochemical monoclonal AMA staining specific for the self-antigen pyruvate dehydrogenase. More recently, PBC was diagnosed in $7 \%$ of patients with pSS. Furthermore, with respect to the $92 \%$ of primary SS patients that were shown to have a positive test for antimitochondrial antibody (AMA), histopathology demonstrated histologic features consistent with $\mathrm{PBC}$, suggesting the importance of AMA screening for SS patients, especially when clinically warranted, such as in the case of elevated alkaline phosphatase (ALP) and aminotransferases [110].

Autoimmune hepatitis is another concern in patients with SS as several studies have reported a higher prevalence of primary autoimmune liver diseases among patients with pSS [111, 112]. Autoimmune hepatitis is found in $1.7 \%$ of patients with primary SS. ANA titers of $>1 / 80$ are associated with the presence of anti-Ro/SSA and anti$\mathrm{La} / \mathrm{SSB}$, whereas titers of $1 / 320$ are associated with presence of anti-smooth muscle and antiribonuclear protein antibodies. SS has also been associated with autoimmune cholangiopathy, including IgG4 and non IgG4 diseases [111, 112].
$\mathrm{HCV}$ infection has an important link with SS; xerostomia is found in up to $35.7 \%$ of $\mathrm{HCV}$ patients. Patients with sicca syndrome and HCV are more expected to have neurological involvement, elevated transaminase levels, rheumatoid factor, and cryoglobulins and less likely to have anti-SSA/SSB antibodies compared with SS patients without HCV [113, 114].

Neoplasia is more common in SS-HCV, including both hepatocellular carcinoma and lymphoproliferative tumors. The most frequent involved organs of lymphoma in SS-HCV patients are the liver and exocrine glands, which are infrequently involved in patients with B cell non-Hodgkin's lymphoma [114].

\section{References}

1. Ebert EC, Hagspiel KD. Gastrointestinal and hepatic manifestations of systemic lupus erythematosus. J Clin Gastroenterol. 2011;45(5):436-41.

2. Fawzy M, Edrees A, Okasha H, El Ashmaui A, Ragab G. Gastrointestinal manifestations in systemic lupus erythematosus. SAGE Journal. 2016:1456-62. https://doi.org/10.1177/0961203316642308.

3. Sultan SM, Loannou Y, et al. A review of gastrointestinal manifestation of systemic lupus erythematosus. Rheumatology. 1999;38:917-32.

4. Chng HH, Tan BE, Teh CL, Lian TY. Major gastrointestinal manifestations in lupus patients in Asia: lupus enteritis, intestinal pseudo-obstruction, and protein-losing gastroenteropathy. Lupus. 2010;19(12):1404-13.

5. Liu Y, Yu J, Oaks Z, et al. Liver injury correlates with biomarkers of autoimmunity and disease activity and represents an organ system involvement in patients with systemic lupus erythematosus. Clin Immunol. 2015;160(2):319-27.

6. Lim DH, Kim YG, Lee D, et al. Immunoglobulin G levels as a prognostic factor for autoimmune hepatitis combined with systemic lupus erythematosus. Arthritis Care Res (Hoboken). 2016;68(7):995-1002.

7. De Santis M, Crotti C. Liver abnormalities in connective tissue diseases. Best Pract Res Clin Gastroenterol. 2013;27:543-51. https://doi. org/10.1016/j.bpg.2013.06.016.

8. Shimizu Y. Liver in systemic disease. World J Gastroenterol. 2008;14(26):4111-9. http://dx.doi. org/10.3748/

9. Abraham S, Begum S, Isenberg D. Hepatic manifestations of autoimmune rheumatic diseases. Ann Rheum Dis. 2004;63:123-9.

10. Schneider A, Merikhi A, Frank BB. Autoimmune disorders: gastrointestinal manifestations and endo- 
scopic findings. Gastrointest Endose Clin N Am. 2006;16(1):133-51

11. Selmi C, De Santis M, Gershwin ME. Liver involvement in subjects with rheumatic disease. Arthritis Research \& Therapy. 2011;13:226. http://arthritisresearch.com/content/13/3/226

12. Goobie GC, Bernatsky S, Ramsey-goldman R, Clarke AE. Malignancies in systemic lupus erythematosus: a 2015 update. Curr Opin Rheumatol. 2015;27(5):454-60.

13. Mao S, Shen H, Zhang J. Systemic lupus erythematosus and malignancies risk. J Cancer Res Clin Oncol. 2016;142(1):253-62.

14. Ebert EC, Hagspiel KD. Gastrointestinal and hepatic manifestations of rheumatoid arthritis. Dig Dis Sci. 2011;56(2):295-302.

15. Nurmohamed MT. Gastrointestinal events in rheumatoid arthritis: time for the lower gastrointestinal tract! J Rheumatol. 2012;39(7):1317-9.

16. Bardou M, Barkun AN. Preventing the gastrointestinal adverse effects of nonsteroidal anti-inflammatory drugs: from risk factor identification to risk factor intervention. Joint Bone Spine. 2010;77:6-123.

17. Cojocaru M, Mihaela I, et al. Extra-articular Manifestations in Rheumatoid Arthritis. A Journal of Clinical Medicine. 2010;5(4)

18. Estrada CA, Lewandowski C, Schubert TT, et al. Esophageal involvement in secondary amyloidosis mimicking achalasia. J Clin Gastroenterol. 1990;12:447-50.

19. Ryutaro M, Masaru M, Tetsuya T, Yoshiro O, Ken Y, Tetsuo A. A case of pneumatosis cystoides intestinalis associated with rheumatoid arthritis, Sjo "gren's syndrome, and abdominal free air. J Jpn Surg Assoc.

20. West SG. Methotrexate hepatotoxicity. Rheum Dis Clin N Am. 1997;23:883-915.

21. Genta MS, Genta RM, Gabay C. Systemic rheumatoid vasculitis:a review. Semin Arthr Rheum. 2006;36:88-98.

22. Fushimi T, Takahashi Y, Kashima Y, et al. Severe protein losing enteropathy with intractable diarrhea due to systemic AAamyloidosis, successfully treated with corticosteroid and octreotide. Amyloid. 2005;12:48-53.

23. Kuroda T, Tanabe N, Sakatsume M, et al. Comparison of gastroduodenal, renal and abdominal fat biopsies for diagnosing amyloidosis in rheumatoid arthritis. Clin Rheum. 2002;21:123-8.

24. Shimoyama M, Ohtahara A, Fukui H, et al. Acute secondary gastrointestinal amyloidosis in a patient with rheumatoid arthritis. Am J Med Sci. 2003;326(3):145-7.

25. Ebert EC. Review article: the gastrointestinal complications of myositis. Aliment Pharmacol Ther. 2010;(3):359-65.

26. Tweezer-zaks N, Ben-horin S, Schiby G, et al. Severe gastrointestinal inflammation in adult dermatomyositis: characterization of a novel clinical association. Am J Med Sci. 2006;332(6):308-13.
27. Sheehan NJ. Dysphagia and other manifestations of oesophageal involvement in the musculoskeletal diseases. Rheumatology (Oxford). 2008;47(6):746-52.

28. Oh TH, Brumfield KA, Hoskin TL, Stolp KA, Murray JA, Bassford JR. Dysphagia in inflammatory myopathy: clinical characteristics, treatment strategies, and outcome in 62 patients. Mayo Clin Proc. 2007;82:441-7.

29. Cherin P, Auperin I, Bussel A, Pourrat J, Herson S. Plasma exchange in polymyositis and dermatomyositis: a multicenter study of 57 cases. Clin ExpRheumatol. 1995;13:270-1.

30. Cherin P, Pelletier S, Teixeira A, et al. Intravenous immunoglobulin for dysphagia of inclusion body myositis. Neurology. 2002;58:326-7.102.

31. Liu LW, Tarnopolsky M, Armstrong D. Injection of botulinum toxin a to the upper esophageal sphincter for oropharyngeal dysphagia in two patients with inclusion body myositis. Can J Gastroenterol. 2004;18:397-9.

32. Hill CL, Zhang Y, Sigurgeirsson B, et al. Frequency of specific cancer types in dermatomyositis and polymyositis: a population-based study. Lancet. 2001;357(9250):96-100.

33. De Santis M, Crotti C, Selmi C. Liver abnormalities in connective tissue diseases. Best Pract Res Clin Gastroenterol. 2013;27(4):543-51.

34. Gyger G, Baron M. Gastrointestinal manifestations of scleroderma: recent progress in evaluation, pathogenesis, and management. Curr Rheumatol Rep. 2012;14(1):22-9.

35. Forbes A, Marie I. Gastrointestinal complications: the most frequent internal complications of systemic sclerosis. Rheumatology (Oxford). 2009;48(Suppl 3):iii36-9.

36. Schmeiser T, Saar P, Jin D, et al. Profile of gastrointestinal involvement in patients with systemic sclerosis. Rheumatol Int. 2012;32(8):2471-8.

37. Marie I, Ducrotte P, Antonietti M, Herve S, Levesque H. Watermelon stomach in systemic sclerosis: its incidence and management. Aliment Pharmacol Ther. 2008;28(4):412-21.

38. Bayraktar Y, Ozaslan E, Van Thiel DH. Gastrointestinal manifestations of Behcet's disease. J Clin Gastroenterol. 2000;30:144-54.

39. Desbois AC, Rautou PE, Biard L, et al. Behcet's disease in budd-chiari syndrome. Orphanet Journal of Rare Diseases. 2014;9:104. https://doi.org/10.1186/ s13023-014-0153-1.

40. Yi SW, Cheon JH, Kim JH, Lee SK, Kim TI, Lee YC, Kim WH. The prevalence and clinical characteristics of esophageal involvement in patients with Behçet's disease: a single center experience in Korea. J Korean Med Sci. 2009;24:52-6. https://doi. org/10.3346/jkms.2009.24.1.52.

41. Houman MH, Ben Ghorbel I, Lamloum M, Khanfir M, Braham A, Haouet S, Sayem N, Lassoued H, Miled M. Esophageal involvement in Behcet's disease. Yonsei Med J. 2002;43:457-60. 
42. Morimoto Y, Tanaka Y, Itoh T, Yamamoto S, Kurihara Y, Nishikawa K. Esophagobronchial fistula in a patient with Behçet's disease:report of a case. Surg Today. 2005;35:671-6. https://doi.org/10.1007/ s00595-004-2975-2.

43. Skef W, Hamilton MJ, Arayssi T. Gastrointestinal Behçet's disease: A review. World J Gastroenterol. 2015;21(13):3801-12. https://doi.org/10.3748/wjg. v21.i13.3801.

44. Bektas M, Altan M, Alkan M, Ormeci N, Soykan I. Manometric evaluation of the esophagus in patients with Behçet's disease. Digestion. 2007;76:192-5. https://doi.org/10.1159/000112645.

45. Ebert EC. Gastrointestinal Manifestations of Behcet's Disease. Dig Dis Sci. 2009;54:201-7. https://doi.org/10.1007/s10620-008-0337-4.

46. Choi IJ, Kim JS, Cha SD, Jung HC, Park JG, Song IS, et al. Long-term clinical course and prognostic factors in intestinal Behcet's disease. Dis Colon Rectum. 2000;43(5):692-700. https://doi. org/10.1007/BF02235590.

47. Lee CR, Kim WH, Cho YS, Kim MH, Kim JH, Park IS, Bang D. Colonoscopic findings in intestinal Behçet's disease. Inflamm Bowel Dis. 2001;7:243-9.

48. Lee SK, Kim BK, Kim TI, Kim WH. Differential diagnosis of intestinal Behçet's disease and Crohn's disease by colonoscopic findings. Endoscopy. 2009;41:9-16. https://doi.org/10.105 5/s-0028-1103481.

49. Chin AB, Kumar AS. Behcet colitis. Clin Colon rectal Surg. 2015;28(2):99-102. https://doi.org/10.105 5/s-0035-1547336.

50. Alkim H, Gürkaynak G, Sezgin O, Oğuz D, Saritaş U, Sahin B. Chronic pancreatitis and aortic pseudoaneurysm in Behçet's disease. Am J Gastroenterol. 2001;96:591-3.

51. Melikoglu M, Kural-Seyahi E, Tascilar K, Yazici $H$. The unique features of vasculitis in Behçet's syndrome. Clin Rev Allergy Immunol. 2008;35:40-6. https://doi.org/10.1007/s12016-007-8064-8.

52. Ebert EC, Hagspiel KD, Nagar M, Schlesinger N. Gastrointestinal involvement in polyarteritis nodosa. Clin Gastroenterol Hepatol. 2008;6(9):960-6.

53. Levine SM, Hellmann DB, Stone JH. Gastrointestinal involvement in polyarteritis nodosa (1986-2000): presentation and outcomes in 24 patients. Am J Med. 2002;112:386-91.

54. Pagnoux C, Mahr A, Cohen P, et al. Presentation and outcome of gastrointestinal involvement in systemic necrotizing vasculitides: analysis of 62 patients with polyarteritis nodosa, microscopic polyangiitis, Wegener granulomatosis, Churg-Strauss syndrome, or rheumatoid arthritis-associated vasculitis. Medicine. 2005;84:115-28.

55. de Carpi JM, Castejon E, Masiques L, et al. Gastrointestinal involvement in pediatric polyarteritis nodosa. J Pediatr Gastroenterol Nutr. 2007;44:274-8.
56. Takeshita S, Nakamura H, Kawakami A, et al. Hepatitis B-related polyarteritis nodosa presenting necrotizing vasculitis in the hepatobiliary system successfully treated with lamivudine,plasmapheresis and glucocorticoid. Intern Med. 2006;45:145-9.

57. Guma M, Lorenzo-Zuniga V, Olive A, et al. Occult liver involvement by polyarteritis nodosa. Clin Rheumatol. 2002;21:184-6.

58. Empen K, Jung MC, Engelhardt D, et al. Successful treatment of acute liver failure due to polyarteritis nodosa. Am J Med. 2002;113:349-51.

59. Hernández-rodríguez J, Alba MA, Prieto-gonzález S, Cid MC. Diagnosis and classification of polyarteritis nodosa. J Autoimmun. 2014;48-49:84-9.

60. Guillevin L, Cohen P, Mahr A, et al. Treatment of polyarteritis nodosa and microscopic polyangiitis with poor prognosis factors: a prospective trial comparing glucocorticoids and six or twelve cyclophosphamide pulses in sixty-five patients. Arthritis Rheum. 2003;49:93-100.

61. Guillevin L, Pagnoux C. Indications of plasma exchanges for systemic vasculitides. Ther Apher Dial. 2003;7:155-60.

62. Pagnoux C, Seror R, Henegar C, Mahr A, Cohen P, Le Guern V, French Vasculitis Study Group, et al. Clinical features and outcomes in 348 patients with polyarteritis nodosa: a systematic retrospective study of patients diagnosed between 1963 and 2005 and entered into the French Vasculitis Study Group Database. Arthritis Rheum. 2010;62:616e26.

63. Craven A, Robson J, Ponte C, Grayson PC, Suppiah $\mathrm{R}$, Judge A, et al. ACR/EULARendorsed study to develop diagnostic and classification criteria for vasculitis (DCVAS). Clin Exp Nephrol. 2013;17:619e21.

64. Masiak A, Zdrojewski Ł, Zdrojewski Z, Bułłopiontecka B, Rutkowski B. Gastrointestinal tract involvement in granulomatosis with polyangiitis. Prz Gastroenterol. 2016;11(4):270-5.

65. Pagnoux C, Mahr A, Cohen P, et al. Presentation and outcome of gastrointestinal involvement in systemic necrotizing vasculitides: analysis of 62 patients with polyarteritis nodosa, microscopic Polyangiitis, Wegener granulomatosis, Churg-Strauss syndrome or rheumatoid arthritis-associated vasculitis [abstract]. Medicine (Baltimore). 2005;84:115-28.

66. Sahin M, Cure E, Goren I, et al. Wegener's granulomatosis presenting with acute renal failure and gastric ulcer. Case Rep Clin Prac Rev. 2006;7:236-9.

67. Deniz K, Őzseker HS, Balas S, et al. Intestinal involvement in Wegener's granulomatosis. J Gastrointestin Liver Dis. 2007;16:329-31.

68. Franco DL, Ruff K, Mertz L, Lam-himlin DM, Heigh R. Eosinophilic granulomatosis with polyangiitis and diffuse gastrointestinal involvement. Case Rep Gastroenterol. 2014;8(3):329-36.

69. Mouthon L, Dunogue B, Guillevin L. Diagnosis and classification of eosinophilic granulomatosis with polyangiitis (formerly named Churg-Strauss syndrome). J Autoimmun. 2014;48-49:99-103. 
70. Kim YB, Choi SW, Park IS, Han JY, Hur YS, Chu YC. Churg-Strauss syndrome with perforating ulcers of the colon. J Korean Med Sci. 2000;15:585-8.

71. Murakami S, Misumi M, Sakata H, Hirayama R, Kubojima Y, Nomura K, Ban S. Churg-Strauss syndrome manifesting as perforation of the small intestine: report of a case. Surg Today. 2004;34:788-92.

72. Esaki M, Matsumoto T, Nakamura S, et al. GI involvement in Henoch-Schfnlein purpura. Gastrointest Endosc. 2002;56:920-3.

73. Chen MJ, Wang TE, Chang WH, et al. Endoscopic findings in a patient with Henoch-Schfnlein purpura. World J Gastroenterol. 2005; 11:2354-6.

74. Pore G. GI lesions in Henoch-Schfnlein purpura. Gastrointest Endosc. 2002;55:283-6.

75. Nakasone H, Hokama A, Fukuchi J, et al. Colonoscopic findings in an adult patient withHenoch-Schfnlein purpura. Gastrointest Endosc. 2000;52:392.

76. Van Erp SJ, Brakenhoff LK, Van Gaalen FA, et al. Classifying Back pain and peripheral joint complaints in inflammatory bowel disease patients: a prospective longitudinal follow-up study. J Crohns Colitis. 2016;10(2):166-75.

77. Arvikar SL, Fisher MC. Inflammatory bowel disease associated arthropathy. Curr Rev Musculoskelet Med. 2011;4:123-31

78. Jacques P, Van Praet L, Carron P, Van Den Bosch F, Elewaut D. Pathophysiology and role of the gastrointestinal system in spondyloarthritides. Rheum Dis Clin N Am. 2012;38(3):569-82.

79. Brakenhoff LKPM, van der Heijde DM, Hommes DW. IBD and arthropathies: a practical approach to its diagnosis and management. Gut. 2011;60:1426e1435. https://doi.org/10.1136/ gut.2010.228866.

80. Orlando A, Renna S, Perricone G, Cottone M. Gastrointestinal lesions associated with Spondyloarthropathies. World J Gastroenterol. 2009;15(20):2443-8. https://doi.org/10.3748/ wjg. 15.2443 .

81. Goldstein JL, Eisen GM, Lewis B, Gralnek IM, Zlotnick S, Fort JG. Video capsule endoscopy to prospectively assess small bowel injury with celecoxib, naproxen plus omeprazole, and placebo. Clin Gastroenterol Hepatol. 2005;3:133-41.

82. Zohar A, Cohen AD, Bitterman H, et al. Gastrointestinal comorbidities in patients with psoriatic arthritis. Clin Rheumatol. 2016; https://doi. org/10.1007/s10067-016-3374-y, 35, 2679

83. de FSP de Oliveira M, Rocha B d O, Duarte GV. Psoriasis:classical and emerging comorbidities. Ann Bras Dermatol. 2015;90:9-20. https://doi. org/10.1590/abd1806-4841.20153038.

84. De Keyser F, Baeten D, Van den Bosch F, De Vos M, Cuvelier C, Mielants H, Veys E. Gut inflammation and spondyloarthropathies. Curr Rheumatol Rep. 2002;4:525-32.
85. Gionchetti P, Rizzello F. IBD: IBD and spondyloarthritis: joint management. Nat Rev Gastroenterol Hepatol. 2016;13(1):9-10.

86. Palm O, Moum B, Ongre A, et al. Prevalence of ankylosing spondylitis and other spondyloarthropathies among patients with inflammatory bowel disease: a population study (the IBSEN study). J Rheumatol. 2002;29:511e15.

87. Steer S, Jones H, Hibbert J, et al. Low back pain, sacroiliitis, and the relationship with HLA-B27 in Crohn's disease. J Rheumatol. 2003;30:518e22.

88. Levine JS, Burakoff R. Extraintestinal manifestations of inflammatory bowel disease. Gastroenterol Hepatol (N Y). 2011;7:235-41.

89. Ebert EC. Gastrointestinal and Hepatic Manifestations of Sjogren Syndrome. J Clin Gastroenterol. 2012;46:25-30.

90. Porter SR, Scully C, Hegarty AM. An update of the etiology and management of xerostomia. Oral Surg Oral Med Oral Pathol Oral Radiol Endod. 2004;97:28-46.

91. Papas AS, Sherrer YS, Charney M, et al. Successful treatment of dry mouth and dry eye symptoms in Sjogren's syndrome patients with oral pilocarpine. A randomized, placebo-controlled, dose-adjustment study. J Clin Rheumatol. 2004;10:169-77.

92. Dawson LJ, Allison HE, Stanbury J, et al. Putative antimuscarinic antibodies cannot be detected in patients with primary Sjogren's syndrome using conventional immunological approaches. Rheumatology. 2004;43:1488-95.

93. Volter F, Fain O, Mathieu E, et al. Esophageal function and Sjfgren's syndrome. Dig Dis Sci. 2004;49:248-53.

94. Turk T, Pirildar T, Tunc E, et al. Manometric assessment of esophageal motility in patients with primary Sjogren's syndrome. Rheumatol Int. 2005;25:246-9.

95. Rogus-Pulia NM, Logemann JA. Effects of reduced saliva production on swallowing in patients with Sjogren's syndrome. Dysphagia. 2011;26:295-303.

96. El Miedany YM, Baddour M, Ahmed I, et al. Sjogren's syndrome: concomitant $\mathrm{H}$. pylori infection and possible correlation with clinical parameters. Joint Bone Spine. 2005;72:135-41.

97. Sorrentino D, Faller G, Devita S, et al. Helicobacter pylori associated antigastric autoantibodies: role in Sjogren's syndrome gastritis. Helicobacter. 2004;9:46-53.

98. Masaki Y, Sugai S. Lymphoproliferative disorders in Sjfgren's syndrome. Autoimmun Rev. 2004;3:175-82.

99. Krogh K, Asmussen K, Stengaard-Pedersen K, et al. Bowel symptoms in patients with primary Sjogren's syndrome. Scand J Rheumatol. 2007;36:407-9.

100. Szodoray P, Barta Z, Lakos G, et al. Coeliac disease in Sjogren's syndrome-a study of 111 Hungarian patients. Rheumatol Int. 2004;24:278-82. 
101. Liden M, Kristjansson G, Valtysdottir S, et al. Gluten sensitivity in patients with primary Sjogren's syndrome. Scand J Gastroenterol. 2007;42:962-7.

102. Katsanos KH, Saougos V, Kosmidou M, et al. Sjogren's syndrome in a patient with ulcerative colitis and primary sclerosing cholangitis: case report and review of the literature. J Crohn Colitis. 2009;3:200-3.

103. Palm A, Moum B, Gran JT. Estimation of Sjogren's syndrome among IBD patients: a six year postdiagnostic prevalence study. Scand J Rheumatol. 2002;31:140-5.

104. Doyle MK. Vasculitis associated with connective tissue disorders. Curr Rheum Reports. 2006;8:312-6.

105. Afzelius P, Fallentin EM, Larsen S, et al. Pancreatic function and morphology in Sjogren's syndrome. Scand J Gastroenterol. 2010;45:752-8.

106. Pickartz T, Pickartz H, Lochs H, et al. Overlap syndrome of autoimmune pancreatitis and cholangitis associated with secondary Sjogren's syndrome. Eur J Gastroenterol Hepatol. 2004;16:1295-9.

107. Bournia VK, Vlachoyiannopoulos PG. Subgroups of Sjogren syndrome patients according to serological profiles. J Autoimmun. 2012;39:15-26.

108. Kaplan MJ, Ike RW. The liver is a common nonexocrine target in primary Sjogren's syndrome: a retrospective review. BMC Gastroenterol. 2002;2:21.

109. Amador-Patarroyo MJ, Arbelaez JG, Mantilla RD, Rodriguez-Rodriguez A, Cardenas-Roldan J, Pineda-Tamayo R, et al. Sjogren's syndrome at the crossroad of polyautoimmunity. J Autoimmun. 2012;39:199-205.

110. Schlenker C, Halterman T, Kowdley KV. Rheumatologic disease and the liver. Clin Liver Dis. 2011;15:153-64.

111. Karp JK, Akpek EK, Anders RA. Autoimmune hepatitis in patients with primary Sjogren's syndrome: a series of two-hundred and two patients. Int J Clin Exp Pathol. 2010;3:582-6.

112. Matsumoto T, Morizane T, Aoki Y, et al. Autoimmune hepatitis in primary Sjogren's syndrome: pathological study of the livers and labial salivary glands in 17 patients with primary Sjogren's syndrome. Pathol Int. 2005;55:70-6.

113. DeVita S, Damato R, DeMarchi G, et al. True primary Sjogren's syndrome in a subset of patients with hepatitis $\mathrm{C}$ infection: a model linking chronic infection to chronic sialadenitis. Isr Med Assoc J. 2002;4:1101-5.

114. Prunoiu C, Georgescu EF, Georgescu M, et al. Sjogren's syndrome associated with chronic hepatitis C-the benefit of the antiviral treatment. Rom J Morph Embry. 2008;49:557-62.

115. Yamashita H, Ueda Y, Kawaguchi H, et al. Systemic lupus erythematosus complicated by Crohn's disease: a case report and literature review. BMC Gastroenterol. 2012;12:174.

116. Malaviya AN, Sharma A, Agarwal D, et al. Acute abdomen in SLE. Int $J$ Rheum Dis. 2011;14(1):98-104.

117. Utiyama SR, Zenatti KB, Nóbrega HA, et al. Rheumatic disease autoantibodies in autoimmune liver diseases. Immunol Investig. 2016;45(6):566-73.

118. Zhang H, Li P, Wu D, et al. Serum IgG subclasses in autoimmune diseases. Medicine (Baltimore). 2015;94(2):e387.

119. Trelle S, Reichenbach S, Wandel S, Hildebrand P, Tschannen B, Villiger PM, et al. Cardiovascular safety of non-steroidal anti-inflammatory drugs: network meta-analysis. BMJ. 2011;342:c7086.

120. Callen JP. Relation between dermatomyositis and polymyositis and cancer. Lancet. 2001;357:85-6.

121. Ben Ghorbel I, Ennaifer R, Lamloum M, Khanfir M, Miled M, Houman MH. Budd-Chiari syndrome associated with Behçet' s disease. Gastroenterol Clin Biol. 2008;32:316-20. https://doi.org/10.1016/j. gcb.2007.12.022.

122. Korkmaz C, Kasifoglu T, Kebapçi M. Budd-Chiari syndrome in the course of Behcet's disease: clinical and laboratory analysis of four cases. Joint Bone Spine. 2007;74:245-8.

123. Fernandes SR, Samara AM, Magalhaes EP, et al. Acute cholecystitis at initial presentation of polyarteritis nodosa. Clin Rheum. 2005;24:625-7.

124. Kim HS, Lee DK, Baik SK, et al. Ileal vasculitis in Henoch-Schfnlein purpura. GastrointestEndosc. 2001;54:493-4.

125. Marie Bailey, William Chapin et al. The effects of vasculitis on the gastrointestinal tract and liver.

126. Binus AM, Han J, Qamar AA, et al. Associated comorbidities in psoriasis and inflammatory bowel disease. J Eur Acad Dermatol Venereol. 2012;26:644-50. https://doi. org/10.1111/j.1468-3083.2011.04153.x.

Open Access This chapter is licensed under the terms of the Creative Commons Attribution 4.0 International License (http://creativecommons.org/licenses/by/4.0/), which permits use, sharing, adaptation, distribution and reproduction in any medium or format, as long as you give appropriate credit to the original author(s) and the source, provide a link to the Creative Commons license and indicate if changes were made.

The images or other third party material in this chapter are included in the chapter's Creative Commons license, unless indicated otherwise in a credit line to the material. If material is not included in the chapter's Creative Commons license and your intended use is not permitted by statutory regulation or exceeds the permitted use, you will need to obtain permission directly from the copyright holder. 